\title{
Geochemical evidence for in situ accumulation of tight gas in the Xujiahe Formation coal measures in the central Sichuan Basin, China
}

\author{
Shengfei Qin ${ }^{\text {* }}$, Yihan Zhang ${ }^{\mathrm{b}}$, Changyi Zhaoa, Zheng Zhou
}

a Research Institute of Petroleum Exploration \& Development (RIPED), PetroChina, No.20 Xueyuan Road, P. O. Box 910, Beijing, 100083, P. R. China

b China University of Petroleum (Beijing), No.18 Fuxue Road, Beijing, 102249, P. R. China

c Lancaster Environment Centre, Lancaster University, Lancaster, LA1 4YQ, United Kingdom

* Corresponding author. Tel.: +86 10 83595689. E-mail address: qsf@petrochina.com.cn (S. Qin).

Abstract: The study of accumulation mechanisms of tight gas has attracted much attention in recent years. One of the focuses is whether natural gas can migrate on a large scale in tight reservoirs. In this work, geochemical parameters of the tight gas reservoirs in the Central Sichuan Basin, China have been studied to characterize the accumulation mechanisms in these fields. Results show that the tight gas accumulation in the Xujiahe Formation in the Central Sichuan is in situ, and natural gas has not experienced large-scale migration. Based on geochemical indicators of natural gas, the gases of Xujiahe Formation in the Central Sichuan Basin originated from the local coal measures of the Xujiahe Formation in horizontal direction with little contribution from the Western Sichuan. In Central Sichuan Basin, there is also no horizontal migration of natural gas in the same formation between adjacent gas fields. Vertically, the Xujiahe Formation is an independent gas generating system and has no relationship with the underlying Mid-Lower Triassic formation and the Jurassic natural gas formation above it. There is a clear distinction in the geochemical characteristics of natural gas between the upper and lower gas reservoirs in the Xujiahe Formation, indicating that there is no obvious vertical migration of natural gas. Geochemical evidence show that there is no large-scale gas migration in the Xujiahe Formation. The tight gas is generated in situ and accumulated in the formation in the Central Sichuan basin.

Key words: Sichuan Basin, Xujiahe Formation, coal measure, tight gas, in situ accumulation, geochemical evidence

\section{Introduction}

With the development of oil and gas explorational technologies, unconventional oil and gas have become the center of focus. In China, tight oil and gas exploration is currently under fast development as a potential oil and gas resources (Jia et al., 2012). Many tight gas fields have been found in Sichuan Basin, Ordos Basin and Tarim Basin in China, and the proven reserves and annual production of tight gas has been increasing with time (Dai et al., 2012a). Together with the unconventional oil and gas exploration, some issues have arisen. Among them, the accumulation mechanisms of tight gas have drawn huge attention. Previous research on the formation of tight gas mainly focused on the evolution of geological conditions in tight gas reservoirs (Che et., 2007; Bian et al., 2009; Tong et al., 2012; Chen et al., 2014; Wei et al. 2016; Wei et al., 2017), formation characterization (Xie et al., 2009) and reservoir geochemistry (Xiao et al., 2008; Dai et al., 2012a, 2012b; Wu et al., 2017). Few studies have been carried out on tight gas migration mechanisms. It is unclear if the natural gas in the tight gas reservoir is generated and accumulated in situ or migrated over a long distance or migrated from sources close to a reservoir. In view of these research questions, this paper takes the coal derived tight gas reservoir in the Xujiahe Formation in the Central 
Sichuan Basin as the study site. The aim is to understand the accumulation processes associated with this tight gas reservoir by characterizing the geochemistry of the natural gas in the reservoir.

The Xujiahe Formation in the Sichuan Basin is mainly composed of a set of coal deposits originated from fluvial, lacustrine and swamp facies (Yang et al., 2009; Xu et al., 2009). It is the first continental strata formation after evolution of the Sichuan basin from marine facies to continental facies. It is commonly developed in the entire Sichuan basin. The formation experienced multiple sedimentary cycles and developed into multiple sets of coal measures interbedded with multiple sets of tight sandstones overlapping each other. Due to the fact that the depositional center of Xujiahe Formation is in the western part of the Sichuan basin, the thickness of coal-bearing source rocks gradually decreases from the west towards the central Sichuan basin (Liu et al., 2005; Chen et al., 2007; Yang et al., 2010), the gas generation intensity of the Xujiahe Formation coal-bearing source rocks is relatively low, less than $20 \times 10^{8} \mathrm{~m}^{3} \mathrm{~km}^{-3}$ in most areas (Fig. 1). This value is the minimum gas-generating intensity to form a reserve of $100 \times 10^{8} \mathrm{~m}^{3}$ in China (Dai et al. 1997). Based on past exploration experience, such a low gas intensity is unlikely to form a large gas field with a reserve of $1000 \times 10^{8} \mathrm{~m}^{3}$. Although the gas intensity is low, but so far a number of large-scale gas fields with proven reserves exceeding $1000 \times 10^{8} \mathrm{~m}^{3}$ have been discovered in the Xujiahe Formation in the Central Sichuan Basin, such as the Xujiahe reservoir in the Anyue gas field, Guang'an gas field, Hechuan gas field, and a series of small and medium gas fields. Some researchers suggested that natural gas in the Xujiahe Formation reservoirs in the Central Sichuan basin mainly comes from the Western Sichuan basin and that the natural gas generated from the thick coal-bearing source rocks in the Xujiahe Formation in the Western Sichuan has laterally migrated long distances to the Central Sichuan Basin. Others proposed that the Xujiahe Formation in the Central Sichuan basin could migrate only short distance because of the strong heterogeneity of the reservoir and the relatively gentle strata (Jiang et al., 2006; Zhao et al., 2011). However there is a lack of geochemical evidence for both hypotheses. There is also a view that the Xujiahe Formation gas reservoir is a "continuous" lithologic gas reservoir formed by evaporative hydrocarbon expulsion of coal-bearing source rocks in a large area (Zou, C., 2009; Yi et al., 2013). It is also suggested that natural gas in the Xujiahe Formation in the Central Sichuan basin is not "large-area contiguous" but dispersed into discrete sheet-type reservoirs (Zhao et al., 2010).

To distinguish between the in-situ and near-field accumulation mechanisms of natural gas in the Xujiahe Formation in the Central Sichuan basin, natural gas migration parameters are used in this study. Results show that natural gas in the Xujiahe Formation originates from coal-bearing source rocks in the Xujiahe Formation itself, with little contribution from other sources. The possibility of natural gas coming from the Xujiahe Formation source rock in the western Sichuan depression has been ruled out. Horizontal and vertical connectivity between the gas reservoirs in different sections in the Xujiahe Formation in the Central Sichuan Basin have also been studied using gas geochemical approaches.

\section{Geological Background}

\subsection{Strata}

The exposed strata in the central Sichuan basin is composed of, from top to bottom, Jurassic (J), Triassic (T), Permian (P), Ordovician (O), Cambrian $(\in)$ and Sinian $(Z)$ stratum, missing Carboniferous (C) and Silurian (S) stratum (Figure 1).

The Upper Jurassic (J3) stratum is mainly a red-brownish mudstone, which forms a good regional seal. The Middle Jurassic (J2) is mainly composed of purple-reddish mudstone, gray-greenish mudstone, silty mudstone and sandstone, which is a good reservoir. Black lacustrine shale developed in lower Jurassic (J1) is not only a good source rock but also a good seal for the underlying gas reservoir.

The Triassic stratum, from top to bottom, includes the Xujiahe Formation $\left(\mathrm{T}_{3} \mathrm{x}\right)$, Leikoupo Formation $\left(T_{2} 1\right)$, Jialingjiang Formation $\left(T_{1 j}\right)$ and Feixianguan Formation $\left(T_{1} f\right)$. The Xujiahe Formation, from bottom 
to top, developed from Xu1 member $\left(T_{3} x^{1}\right)$ to Xu6 member $\left(T_{3} x^{6}\right)$, with $\mathrm{Xu}\left(\mathrm{T}_{3} \mathrm{X}^{1}\right), \mathrm{Xu} 3\left(\mathrm{~T}_{3} \mathrm{x}^{3}\right)$ and $\mathrm{Xu} 5$ $\left(\mathrm{T}_{3} \mathrm{X}^{5}\right)$ members dominated by coal and dark mudstone, interbedded with thin layers of sandstone, which form main hydrocarbon source rock. The $\mathrm{Xu} 2\left(\mathrm{~T}_{3} \mathrm{x}^{2}\right)$, Xu4 $\left(\mathrm{T}_{3} \mathrm{x}^{4}\right)$ and $\mathrm{Xu} 6\left(\mathrm{~T}_{3} \mathrm{x}^{6}\right)$ members are predominantly white and gray fine-medium sandstone reservoirs (Figure 1). The Leikoupo Formation is dominated by dolomite, intercalated with gypsum and thin gray-black shale, which forms the region's high-quality seal. The dolomite within the Leikoupo Formation is also a good reservoir; The Jialingjiang Formation is limestone interbedded with dolomite and gypsum layer. It developed well as both the reservoir and cap rock. The Feixianguan Formation is mainly composed of oolitic and limestone with dissolved pores, which form high-quality regional reservoir rocks.

The upper Permian stratum is dominated by bioclastic limestone, reef limestone and dolomite. The transitional coal measure and limestone are developed in the middle part of the Permian stratum. The limestone and dolomitic limestone form the lower part of the Permian stratum. The bottom of Permian stratum is composed of thin-layer shale, sandstone and limestone. The Ordovician stratum is dominated by biogenic limestone and oolitic limestone and has been denuded in the upper part of the stratum. The Cambrian stratum is mainly limestone and dolomite, the lower part of the stratum has been developed into thick gray-black shale, which forms high-quality source rock. The Sinian stratum is dominated by dolomite intercalated with thin gray-black shale.

\subsection{Structure}

According to the tectonic division of the Sichuan Basin, the central Sichuan basin is also termed "the gentle tectonic zone in the middle of Sichuan ". Sedimentary cap rocks in the central Sichuan basin are thin and stressed weakly. The slipping layer has not been developed. It forms a gentle slope structure (Wang et al., 2005). The Central Sichuan basin was uplifted to land by Indosinian movement in early Middle Triassic. Since the late Triassic continental deposition began to develop and formed the Xujiahe Formation, which is a sedimentary assemblage of coal-bearing source rocks interbedded with sandstones.

In the Early Jurassic, lacustrine sediments deposited in the Sichuan Basin under a stable environment, forming the lacustrine source rocks. In The Middle Jurassic, rapid deposition from rivers and shallow lakes forms the main sedimentary period of the continental basin. In the Late Jurassic, deposition from turbulent lake and fluvial sediments lasted until the end of Jurassic. Due to intensive uplift and denudation caused by Himalayan movement, the upper Jurassic and strata above it were missing in most areas. Thickness of the eroded stratum is about $2500 \mathrm{~m}$ (Chen, et al., 2007). Stratum uplift and denudation contributed to lower formation temperature and pressure, and hindered further hydrocarbon generation by organic matter in the source rocks. The Himalayan movement uplifted the central Sichuan basin as a whole. There were no large fault systems created. This is favorable to the later natural gas preservation.

\subsection{Gas Reservoir Types}

Gas reservoirs in the Xujiahe Formation in the Central Sichuan basin are widely distributed covering a large area. They are found in $\mathrm{T} 3 \mathrm{x}^{2}, \mathrm{~T} 3 \mathrm{x}^{4}$ and $\mathrm{T} 3 \mathrm{x}^{6}$ longitudinally. Currently the structural high is in the south part of the basin with north part as a structural low to form a large regional monocline. Tectonic stress of Hechuan, Guang'an, Moxi-Longniansi, Nanchong and Bajiaochang areas are relatively strong. Most of the structures are relatively gentle and the gas reservoirs are dominated by structural-lithologic gas reservoirs (Wang et al., 2005; Xu et al., 2009).

According to statistics of tens of thousands of physical data, reservoir properties of the Xujiahe Formation are poor. Porosity is ranging between $4-8 \%$ and permeability distribution is within the range of $0.01-1 \mathrm{mD}$, which suggest that reservoirs can be categorized as low porosity-low permeability and ultra-low porosity and ultra-low permeability reservoirs (Yang et al., 2010; Zhang et al., 2011). Such kind of low porosity and low permeability tight sandstone reservoirs require horizontal fracturing and other stimulation measures to obtain industrial gas flow (Shanley et al., 2004). The gas reservoirs also have high water 
content and the reserve abundance is $1-3 \times 10^{8} \mathrm{~m}^{-3}$, which belongs to medium-low abundance high water gas reservoirs (Zhao et al., 2010).

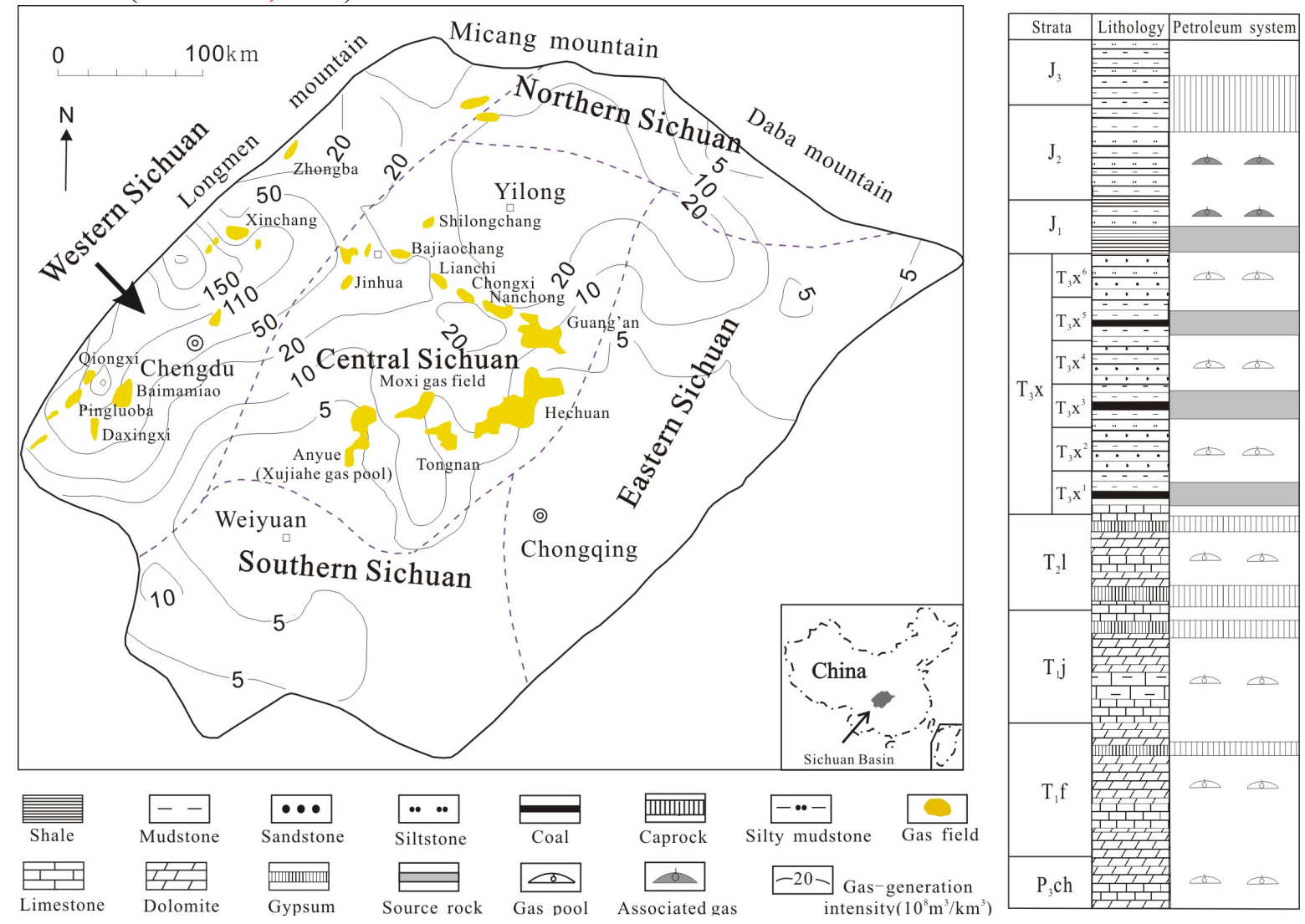

Fig. 1. Map of gas field distribution in the Xujiahe Formation and stratigraphic column in the Central Sichuan basin.

\section{Sampling and analytical methods}

\subsection{Sample collection}

Natural gas samples were taken from the Middle-Upper Jurassic, Upper Triassic Xujiahe Formation, Middle Triassic Leikoupo Formation, Lower Triassic Jialingjiang Formation in the Central Sichuan Basin, and the Xujiahe Formation reservoirs in the Western Sichuan Basin. To eliminate the interferences of external factors and ensure representativeness of the natural gas in these reservoirs, all samples were collected from wells with long-term normal production without application of de-foaming or any other chemical agents recently.

Gas samples in reservoirs were taken at the wellheads by using steel cylinders. To take such samples, the pressure gauge was dismantled before connecting the steel cylinder with sampling tubing. Prior to taking samples, wellhead natural gas was used to flush the steel cylinder thoroughly for about 3 minutes. The sampling steel cylinder was then filled with natural gas equilibrated to the wellhead pressure.

\subsection{Analytical methods}

Samples were analyzed in the Key laboratory of the Research Institute of Exploration and Development of PetroChina. Natural gas compositions were determined using an Agilent $6890 \mathrm{~N}$ gas chromatograph (GC) with $\mathrm{He}$ and $\mathrm{N}_{2}$ as the carrier gases. Double thermal conductivity detectors (TCD) and a $30 \mathrm{~m} \times 0.25 \mathrm{~mm} \times 0.25 \mu \mathrm{m}$ quartz capillary column were used. The inlet temperature was $150{ }^{\circ} \mathrm{C}$, and the TCD temperature was $200{ }^{\circ} \mathrm{C}$. The initial oven temperature was maintained at $40{ }^{\circ} \mathrm{C}$ for $7.5 \mathrm{~min}$ isothermally, then rose from $40{ }^{\circ} \mathrm{C}$ to $90{ }^{\circ} \mathrm{C}$ at $15{ }^{\circ} \mathrm{C} / \mathrm{min}$, and finally rose from $90{ }^{\circ} \mathrm{C}$ to $180{ }^{\circ} \mathrm{C}$ at $6{ }^{\circ} \mathrm{C} / \mathrm{min}$. 
The on-line analysis was conducted for the measurement of carbon isotopic compositions with a MAT 253 gas isotopic mass spectrometer. Natural gas samples were separated to methane, ethane, propane, butane and $\mathrm{CO}_{2}$ through the chromatography column of a SRI 8610C gas chromatograph. They were then transferred into combustion furnace by carrier gas $(\mathrm{He})$ and oxidized into $\mathrm{CO}_{2}$ by $\mathrm{CuO}$ at $850{ }^{\circ} \mathrm{C}$. All of the converted species were transferred by carrier gas (He) into MS to measure the isotopic compositions. Dual inlet analysis was performed with international measurement standard of NBS-19 $\mathrm{CO}_{2}$ $\left(\delta^{13} \mathrm{C}_{\mathrm{VPDB}}=1.95 \pm 0.04 \%\right.$, International Atomic Energy Agency, 1995) and the stable carbon isotopic values were reported in the $\delta$ notation in per mil (\%) relative to the Peedee belemnite standard (VPDB). Reproducibility and accuracy were estimated to be $\pm 0.2 \%$ with respect to VPDB standard.

\section{Result}

Many researchers have carried out studies on the natural gas geochemistry in the Xujiahe Formation in the Sichuan basin. They have reached similar conclusions that the gas in the formation is mainly coal-type gas (Xiao et al., 2008; Dai et al., 2012a, 2012b; Wu et al., 2017). Here we are not repeating similar research in this paper, but focusing on some geochemical features that were not discussed in previous work.

Table 1

Molecular composition of natural gases in Xujiahe Formation in the Central Sichuan Basin

\begin{tabular}{|c|c|c|c|c|c|c|c|c|c|c|c|c|c|c|c|c|}
\hline \multirow{2}{*}{ Gas field } & \multirow{2}{*}{ Well } & \multirow{2}{*}{ Strata } & \multicolumn{11}{|c|}{ Main molecular composition (\%) } & \multicolumn{3}{|c|}{ Calculated parameters } \\
\hline & & & $\mathrm{N}_{2}$ & $\mathrm{CO}_{2}$ & $\mathrm{C}_{1}$ & $\mathrm{C}_{2}$ & $\mathrm{C}_{3}$ & $\mathrm{iC}_{4}$ & $\mathrm{nC}_{4}$ & $\mathrm{iC}_{5}$ & $\mathrm{nC}_{5}$ & $\mathrm{C}_{6+}$ & $\mathrm{C}_{1+}$ & $\mathrm{iC}_{4} / \mathrm{nC}_{4}$ & $\mathrm{iC}_{5} / \mathrm{nC}_{5}$ & $\mathrm{C}_{1} / \mathrm{C}_{1+}$ \\
\hline \multirow[t]{15}{*}{ Hechuan } & Hechuan 1 & $\mathrm{~T}_{3} \mathrm{X}^{2}$ & 1.42 & 0.12 & 88.84 & 6.39 & 1.58 & 0.39 & 0.27 & 0.16 & 0.07 & 0.33 & 98.02 & 1.45 & 2.43 & 0.906 \\
\hline & Hechuan 101 & $\mathrm{~T}_{3} \mathrm{X}^{2}$ & 0.08 & 0.19 & 93.29 & 4.54 & 0.64 & 0.12 & 0.07 & 0.03 & 0.01 & 0.11 & 98.82 & 1.77 & 2.90 & 0.944 \\
\hline & Hechuan 102 & $\mathrm{~T}_{3} \mathrm{X}^{2}$ & 0.00 & 0.23 & 87.05 & 5.65 & 1.43 & 0.36 & 0.24 & 0.15 & 0.06 & 1.21 & 96.15 & 1.54 & 2.30 & 0.905 \\
\hline & Hechuan 105 & $\mathrm{~T}_{3} \mathrm{X}^{2}$ & 0.16 & 0.22 & 91.41 & 5.71 & 1.01 & 0.21 & 0.15 & 0.07 & 0.03 & 0.22 & 98.81 & 1.39 & 2.18 & 0.925 \\
\hline & Hechuan 106 & $\mathrm{~T}_{3} \mathrm{X}^{2}$ & 0.00 & 0.16 & 90.15 & 5.95 & 1.48 & 0.38 & 0.29 & 0.18 & 0.08 & 0.57 & 99.06 & 1.29 & 2.33 & 0.910 \\
\hline & Hechuan 110 & $\mathrm{~T}_{3} \mathrm{X}^{2}$ & 0.00 & 0.03 & 92.83 & 4.43 & 0.76 & 0.18 & 0.11 & 0.07 & 0.03 & 0.24 & 98.65 & 1.69 & 2.44 & 0.941 \\
\hline & Hechuan 112 & $\mathrm{~T}_{3} \mathrm{X}^{2}$ & 0.00 & 0.19 & 90.47 & 6.05 & 1.42 & 0.36 & 0.23 & 0.13 & 0.05 & 0.24 & 98.96 & 1.56 & 2.54 & 0.914 \\
\hline & Hechuan 117 & $\mathrm{~T}_{3} \mathrm{X}^{2}$ & 0.04 & 0.23 & 90.43 & 5.88 & 1.41 & 0.40 & 0.27 & 0.14 & 0.05 & 0.16 & 98.74 & 1.49 & 2.77 & 0.916 \\
\hline & Hechuan 118 & $\mathrm{~T}_{3} \mathrm{x}^{2}$ & 0.01 & 0.1 & 89.41 & 6.28 & 1.57 & 0.42 & 0.31 & 0.19 & 0.08 & 0.83 & 99.10 & 1.37 & 2.26 & 0.902 \\
\hline & Hechuan 119 & $\mathrm{~T}_{3} \mathrm{x}^{2}$ & 0.10 & 0.12 & 89.93 & 5.64 & 1.23 & 0.37 & 0.26 & 0.20 & 0.09 & 0.61 & 98.33 & 1.46 & 2.36 & 0.915 \\
\hline & Hechuan 124 & $\mathrm{~T}_{3} \mathrm{X}^{2}$ & 0.02 & 0.42 & 89.78 & 5.83 & 1.46 & 0.43 & 0.33 & 0.25 & 0.11 & 0.57 & 98.75 & 1.31 & 2.33 & 0.909 \\
\hline & Hechuan 4 & $\mathrm{~T}_{3} \mathrm{X}^{2}$ & 0.81 & 0.06 & 88.88 & 6.21 & 1.71 & 0.67 & 0.49 & 0.41 & 0.17 & 0.00 & 98.54 & 1.37 & 2.41 & 0.902 \\
\hline & Hechuan 5 & $\mathrm{~T}_{3} \mathrm{x}^{2}$ & 0.15 & 0.09 & 92.96 & 5.08 & 0.96 & 0.26 & 0.17 & 0.09 & 0.03 & 0.00 & 99.55 & 1.53 & 3.00 & 0.934 \\
\hline & Hechuan 6 & $\mathrm{~T}_{3} \mathrm{X}^{2}$ & 0.98 & 0.27 & 88.23 & 6.97 & 1.84 & 0.45 & 0.33 & 0.18 & 0.08 & 0.47 & 98.55 & 1.34 & 2.33 & 0.895 \\
\hline & Hechuan 7 & $\mathrm{~T}_{3} \mathrm{x}^{2}$ & 0.69 & 0.39 & 90.82 & 5.88 & 1.35 & 0.33 & 0.24 & 0.12 & 0.05 & 0.00 & 98.79 & 1.38 & 2.40 & 0.919 \\
\hline \multirow[t]{10}{*}{ Tongnan } & Tongnan 001-1 & $\mathrm{T}_{3} \mathrm{X}^{2}$ & 0.02 & 0.31 & 88.07 & 7.22 & 2.05 & 0.46 & 0.40 & 0.20 & 0.09 & 0.41 & 98.90 & 1.14 & 2.25 & 0.891 \\
\hline & Tongnan 001-5 & $\mathrm{T}_{3} \mathrm{X}^{2}$ & 0.05 & 0.25 & 87.02 & 7.28 & 2.25 & 0.58 & 0.51 & 0.28 & 0.13 & 0.41 & 98.47 & 1.14 & 2.10 & 0.884 \\
\hline & Tongnan 102 & $\mathrm{~T}_{3} \mathrm{X}^{2}$ & 1.15 & 0.53 & 85.77 & 8.27 & 2.60 & 0.58 & 0.51 & 0.22 & 0.10 & 0.20 & 98.25 & 1.12 & 2.13 & 0.873 \\
\hline & Tongnan 107 & $\mathrm{~T}_{3} \mathrm{x}^{2}$ & 0.06 & 0.29 & 87.94 & 7.49 & 2.02 & 0.44 & 0.37 & 0.17 & 0.08 & 0.29 & 98.80 & 1.19 & 2.25 & 0.890 \\
\hline & Tongnan 108 & $\mathrm{~T}_{3} \mathrm{X}^{2}$ & 0.00 & 0.3 & 84.73 & 8.43 & 2.80 & 0.58 & 0.60 & 0.27 & 0.15 & 0.50 & 98.05 & 0.97 & 1.82 & 0.864 \\
\hline & Tongnan 110 & $\mathrm{~T}_{3} \mathrm{X}^{2}$ & 0.00 & 0.43 & 86.82 & 7.35 & 2.05 & 0.49 & 0.45 & 0.30 & 0.16 & 1.10 & 98.71 & 1.09 & 1.89 & 0.880 \\
\hline & Tongnan 113 & $\mathrm{~T}_{3} \mathrm{x}^{2}$ & 0.10 & 0.63 & 82.29 & 8.26 & 2.89 & 0.76 & 0.69 & 0.36 & 0.17 & 0.41 & 95.82 & 1.11 & 2.06 & 0.859 \\
\hline & Tongnan 114 & $\mathrm{~T}_{3} \mathrm{x}^{2}$ & 0.00 & 0.37 & 83.98 & 7.98 & 2.98 & 0.61 & 0.70 & 0.32 & 0.21 & 0.76 & 97.53 & 0.88 & 1.54 & 0.861 \\
\hline & Tongnan 3 & $\mathrm{~T}_{3} \mathrm{x}^{2}$ & 2.00 & 0.32 & 78.91 & 8.76 & 3.78 & 1.25 & 1.43 & 1.13 & 0.64 & 1.63 & 97.52 & 0.88 & 1.76 & 0.809 \\
\hline & Tongnan 6 & $\mathrm{~T}_{3} \mathrm{x}^{2}$ & 0.05 & 0.37 & 88.56 & 6.62 & 1.78 & 0.39 & 0.35 & 0.18 & 0.09 & 0.56 & 98.52 & 1.14 & 2.01 & 0.899 \\
\hline \multirow[t]{11}{*}{ Nanchong } & Xi 20 & $\mathrm{~T}_{3} \mathrm{x}^{2}$ & 0.88 & 0.35 & 89.82 & 5.72 & 2.07 & 0.40 & 0.43 & 0.12 & 0.06 & 0.07 & 98.70 & 0.93 & 1.95 & 0.910 \\
\hline & Xi 35-1 & $\mathrm{T}_{3} \mathrm{X}^{2}$ & 1.48 & 0.17 & 86.77 & 6.08 & 1.88 & 0.58 & 0.47 & 0.35 & 0.18 & 2.01 & 98.32 & 1.25 & 2.01 & 0.883 \\
\hline & Xi 13-1 & $\mathrm{T}_{3} \mathrm{x}^{4}$ & 1.50 & 1.69 & 85.00 & 6.64 & 2.74 & 0.52 & 0.65 & 0.26 & 0.17 & 0.69 & 96.66 & 0.79 & 1.56 & 0.879 \\
\hline & Xi 32 & $\mathrm{~T}_{3} \mathrm{x}^{4}$ & 3.99 & 0.21 & 82.72 & 7.20 & 3.30 & 0.63 & 0.84 & 0.31 & 0.21 & 0.54 & 95.76 & 0.76 & 1.48 & 0.864 \\
\hline & Xi 48 & $\mathrm{~T}_{3} \mathrm{x}^{4}$ & 1.32 & 0.87 & 88.18 & 6.07 & 2.09 & 0.42 & 0.47 & 0.18 & 0.11 & 0.20 & 97.73 & 0.89 & 1.62 & 0.902 \\
\hline & Xi 56 & $\mathrm{~T}_{3} \mathrm{x}^{4}$ & 0.15 & 0.66 & 88.00 & 6.22 & 2.32 & 0.48 & 0.53 & 0.20 & 0.12 & 0.34 & 98.20 & 0.89 & 1.66 & 0.896 \\
\hline & Xi 57 & $T_{3} x^{4}$ & 0.94 & 0.1 & 83.78 & 8.55 & 3.60 & 0.75 & 1.05 & 0.41 & 0.23 & & 97.97 & 0.72 & 1.58 & 0.855 \\
\hline & Xi 58 & $\mathrm{~T}_{3} \mathrm{x}^{4}$ & 1.42 & 0.82 & 85.62 & 7.08 & 2.84 & 0.52 & 0.60 & 0.19 & 0.13 & 0.63 & 97.61 & 0.86 & 1.54 & 0.877 \\
\hline & Xi 62 & $\mathrm{~T}_{3} \mathrm{x}^{4}$ & 2.14 & 0.38 & 82.33 & 7.58 & 3.57 & 0.74 & 1.12 & 0.49 & 0.38 & 1.25 & 97.45 & 0.66 & 1.31 & 0.845 \\
\hline & Xi 64 & $T_{3} x^{4}$ & 0.76 & 0.28 & 88.92 & 6.03 & 2.16 & 0.44 & 0.48 & 0.20 & 0.13 & 0.53 & 98.88 & 0.91 & 1.58 & 0.899 \\
\hline & Xi 65 & $\mathrm{~T}_{3} \mathrm{x}^{4}$ & 1.65 & 0.24 & 87.71 & 6.22 & 2.32 & 0.44 & 0.54 & 0.21 & 0.14 & 0.50 & 98.08 & 0.81 & 1.54 & 0.894 \\
\hline
\end{tabular}




\begin{tabular}{|c|c|c|c|c|c|c|c|c|c|c|c|c|c|c|c|c|}
\hline & Xi 69 & $\mathrm{~T}_{3} \mathrm{x}^{4}$ & 0.02 & 0.74 & 89.62 & 5.27 & 1.89 & 0.41 & 0.46 & 0.18 & 0.12 & 0.36 & 98.31 & 0.88 & 1.59 & 0.912 \\
\hline & Xi 71 & $\mathrm{~T}_{3} \mathrm{x}^{4}$ & 0.16 & 0.73 & 88.66 & 5.99 & 2.00 & 0.42 & 0.47 & 0.18 & 0.12 & 0.45 & 98.29 & 0.90 & 1.59 & 0.902 \\
\hline & Xi 72 & $\mathrm{~T}_{3} \mathrm{x}^{4}$ & 0.20 & 0.26 & 87.31 & 6.68 & 2.83 & 0.57 & 0.72 & 0.30 & 0.19 & 0.90 & 99.50 & 0.79 & 1.56 & 0.877 \\
\hline & Xi 73X & $\mathrm{T}_{3} \mathrm{x}^{4}$ & 0.88 & 0.70 & 79.01 & 6.24 & 3.49 & 1.43 & 2.26 & 1.86 & 1.29 & 2.83 & 98.40 & 0.63 & 1.44 & 0.803 \\
\hline \multirow[t]{45}{*}{ Guang'an } & Guang'an 003-2 & $\mathrm{T}_{3} \mathrm{x}^{4}$ & 0.00 & 0.31 & 93.97 & 3.71 & 0.63 & 0.13 & 0.08 & 0.04 & 0.01 & 0.13 & 98.70 & 1.61 & 2.50 & 0.952 \\
\hline & Guang'an 106 & $\mathrm{~T}_{3} \mathrm{x}^{4}$ & 0.03 & 0.34 & 93.25 & 4.32 & 0.75 & 0.14 & 0.10 & 0.04 & 0.02 & 0.16 & 98.78 & 1.50 & 2.32 & 0.944 \\
\hline & Guang'an 112 & $\mathrm{~T}_{3} \mathrm{x}^{4}$ & 0.92 & 0.41 & 92.71 & 4.52 & 0.86 & 0.15 & 0.12 & 0.05 & 0.02 & 0.19 & 98.62 & 1.26 & 2.38 & 0.940 \\
\hline & Guang'an 113 & $\mathrm{~T}_{3} \mathrm{x}^{4}$ & 0.90 & 0.36 & 93.51 & 4.01 & 0.70 & 0.14 & 0.10 & 0.05 & 0.02 & 0.14 & 98.65 & 1.44 & 2.65 & 0.948 \\
\hline & Guang'an 114 & $\mathrm{~T}_{3} \mathrm{x}^{4}$ & 0.35 & 0.27 & 94.72 & 3.84 & 0.55 & 0.08 & 0.06 & 0.02 & 0.01 & 0.07 & 99.35 & 1.33 & 2.00 & 0.953 \\
\hline & Guang'an 116 & $T_{3} x^{4}$ & 0.83 & 0.20 & 93.07 & 4.60 & 0.77 & 0.16 & 0.10 & 0.05 & 0.02 & 0.16 & 98.93 & 1.64 & 2.72 & 0.941 \\
\hline & Guang'an 121 & $\mathrm{~T}_{3} \mathrm{x}^{4}$ & 1.17 & 0.29 & 92.49 & 4.67 & 0.83 & 0.18 & 0.12 & 0.06 & 0.03 & 0.08 & 98.45 & 1.45 & 2.32 & 0.939 \\
\hline & Guang'an 122 & $\mathrm{~T}_{3} \mathrm{x}^{4}$ & 1.42 & 0.27 & 92.21 & 4.59 & 0.76 & 0.19 & 0.11 & 0.06 & 0.02 & 0.14 & 98.07 & 1.76 & 2.77 & 0.940 \\
\hline & Guang'an 123 & $T_{3} x^{4}$ & 1.05 & 0.37 & 91.85 & 5.11 & 0.95 & 0.22 & 0.17 & 0.09 & 0.03 & 0.07 & 98.49 & 1.26 & 2.78 & 0.933 \\
\hline & Guang'an 125 & $\mathrm{~T}_{3} \mathrm{x}^{4}$ & 1.37 & 0.24 & 92.11 & 4.73 & 0.90 & 0.21 & 0.13 & 0.07 & 0.02 & 0.09 & 98.26 & 1.63 & 2.96 & 0.937 \\
\hline & Guang'an 126 & $T_{3} x^{4}$ & 0.75 & 0.33 & 93.32 & 4.43 & 0.75 & 0.15 & 0.10 & 0.05 & 0.02 & 0.09 & 98.90 & 1.49 & 2.42 & 0.944 \\
\hline & Guang'an 127 & $\mathrm{~T}_{3} \mathrm{x}^{4}$ & 1.29 & 0.33 & 92.21 & 4.76 & 0.82 & 0.17 & 0.10 & 0.04 & 0.01 & 0.05 & 98.16 & 1.63 & 3.33 & 0.939 \\
\hline & Guang'an 128 & $\mathrm{~T}_{3} \mathrm{x}^{4}$ & 0.14 & 0.37 & 93.01 & 4.38 & 0.76 & 0.15 & 0.10 & 0.05 & 0.02 & 0.16 & 98.63 & 1.51 & 2.30 & 0.943 \\
\hline & Guang'an 130 & $\mathrm{~T}_{3} \mathrm{x}^{4}$ & 1.09 & 0.26 & 92.37 & 4.94 & 0.96 & 0.16 & 0.11 & 0.03 & 0.01 & 0.05 & 98.62 & 1.47 & 2.70 & 0.937 \\
\hline & Guang'an 131 & $T_{3} x^{4}$ & 0.15 & 0.24 & 93.16 & 4.39 & 0.74 & 0.14 & 0.10 & 0.04 & 0.01 & 0.08 & 98.66 & 1.48 & 2.69 & 0.944 \\
\hline & Guang'an 133 & $\mathrm{~T}_{3} \mathrm{x}^{4}$ & 0.92 & 0.92 & 92.30 & 4.20 & 0.68 & 0.13 & 0.09 & 0.05 & 0.02 & 0.50 & 97.98 & 1.44 & 2.08 & 0.942 \\
\hline & Guang'an 134 & $T_{3} x^{4}$ & 0.19 & 0.07 & 92.99 & 4.45 & 0.68 & 0.13 & 0.08 & 0.03 & 0.01 & 0.07 & 98.43 & 1.68 & 2.55 & 0.945 \\
\hline & Guang'an 136 & $\mathrm{~T}_{3} \mathrm{x}^{4}$ & 0.89 & 0.28 & 92.15 & 4.94 & 0.94 & 0.22 & 0.14 & 0.07 & 0.03 & 0.19 & 98.68 & 1.56 & 2.39 & 0.934 \\
\hline & Guang'an 142 & $T_{3} x^{4}$ & 0.14 & 0.33 & 92.33 & 4.69 & 0.90 & 0.21 & 0.14 & 0.08 & 0.04 & 0.30 & 98.69 & 1.56 & 2.37 & 0.936 \\
\hline & Guang'an 143 & $\mathrm{~T}_{3} \mathrm{x}^{4}$ & 0.20 & 0.29 & 93.10 & 3.91 & 0.62 & 0.11 & 0.07 & 0.03 & 0.01 & 0.19 & 98.04 & 1.61 & 2.58 & 0.950 \\
\hline & Guang'an 144 & $\mathrm{~T}_{3} \mathrm{x}^{4}$ & 0.03 & 0.30 & 93.35 & 4.27 & 0.70 & 0.15 & 0.09 & 0.04 & 0.02 & 0.13 & 98.74 & 1.68 & 2.56 & 0.945 \\
\hline & Guang'an 145 & $\mathrm{~T}_{3} \mathrm{x}^{4}$ & 0.15 & 1.60 & 94.06 & 2.32 & 0.23 & 0.04 & 0.03 & 0.01 & 0.01 & 0.09 & 96.79 & 1.48 & 2.00 & 0.972 \\
\hline & Guang'an 16 & $\mathrm{~T}_{3} \mathrm{x}^{4}$ & 0.64 & 0.41 & 92.32 & 4.94 & 1.05 & 0.24 & 0.18 & 0.07 & 0.03 & 0.07 & 98.89 & 1.35 & 2.67 & 0.934 \\
\hline & Guang'an 17 & $\mathrm{~T}_{3} \mathrm{x}^{4}$ & 0.61 & 0.46 & 93.10 & 4.50 & 0.88 & 0.19 & 0.13 & 0.06 & 0.02 & 0.05 & 98.92 & 1.42 & 2.62 & 0.941 \\
\hline & Guang'an 20 & $\mathrm{~T}_{3} \mathrm{x}^{4}$ & 0.22 & 0.27 & 91.89 & 4.68 & 0.97 & 0.23 & 0.17 & 0.11 & 0.05 & 0.51 & 98.59 & 1.35 & 2.19 & 0.932 \\
\hline & Guang'an 3 & $T_{3} x^{4}$ & 1.99 & 0.35 & 93.73 & 3.31 & 0.42 & 0.07 & 0.04 & 0.02 & 0.01 & 0.00 & 97.60 & 1.73 & 2.83 & 0.960 \\
\hline & Guang'an 002-21 & $T_{3} x^{6}$ & 0.84 & 0.63 & 89.46 & 6.26 & 1.68 & 0.33 & 0.35 & 0.13 & 0.07 & 0.17 & 98.45 & 0.96 & 1.83 & 0.909 \\
\hline & Guang'an 002-X77 & $\mathrm{T}_{3} \mathrm{x}^{6}$ & 0.01 & 0.44 & 89.37 & 5.97 & 1.72 & 0.35 & 0.36 & 0.16 & 0.09 & 0.43 & 98.44 & 0.96 & 1.72 & 0.908 \\
\hline & Guang'an 103 & $\mathrm{~T}_{3} \mathrm{x}^{6}$ & 0.72 & 0.64 & 87.40 & 7.59 & 2.10 & 0.40 & 0.37 & 0.14 & 0.07 & 0.15 & 98.22 & 1.07 & 2.03 & 0.890 \\
\hline & Guang'an 104 & $\mathrm{~T}_{3} \mathrm{x}^{6}$ & 0.83 & 0.63 & 88.85 & 6.56 & 1.77 & 0.35 & 0.35 & 0.15 & 0.07 & 0.36 & 98.45 & 1.00 & 2.03 & 0.902 \\
\hline & Guang'an 105 & $\mathrm{~T}_{3} \mathrm{x}^{6}$ & 0.92 & 0.54 & 89.20 & 6.10 & 1.81 & 0.39 & 0.40 & 0.16 & 0.08 & 0.27 & 98.40 & 0.96 & 2.11 & 0.907 \\
\hline & Guang'an 109 & $T_{3} x^{6}$ & 0.71 & 0.57 & 89.16 & 6.13 & 1.72 & 0.34 & 0.34 & 0.14 & 0.07 & 0.29 & 98.19 & 1.01 & 2.09 & 0.908 \\
\hline & Guang'an 110 & $T_{3} x^{6}$ & 0.81 & 0.39 & 89.81 & 6.08 & 1.65 & 0.33 & 0.32 & 0.13 & 0.07 & 0.33 & 98.71 & 1.02 & 1.97 & 0.910 \\
\hline & Guang'an 111 & $T_{3} x^{6}$ & 0.06 & 0.36 & 89.29 & 6.39 & 1.79 & 0.35 & 0.36 & 0.15 & 0.09 & 0.29 & 98.71 & 0.97 & 1.61 & 0.905 \\
\hline & Guang'an 112 & $T_{3} x^{6}$ & 1.61 & 0.33 & 89.66 & 5.92 & 1.39 & 0.24 & 0.26 & 0.11 & 0.06 & 0.18 & 97.82 & 0.90 & 1.98 & 0.917 \\
\hline & Guang'an 114 & $\mathrm{~T}_{3} \mathrm{x}^{6}$ & 2.30 & 0.35 & 90.61 & 4.99 & 0.98 & 0.143 & 0.149 & 0.054 & 0.022 & 0.091 & 97.04 & 0.96 & 2.45 & 0.934 \\
\hline & Guang'an 115 & $\mathrm{~T}_{3} \mathrm{x}^{6}$ & 2.23 & 0.19 & 88.05 & 6.58 & 1.74 & 0.36 & 0.35 & 0.13 & 0.07 & 0.23 & 97.51 & 1.02 & 1.94 & 0.903 \\
\hline & Guang'an 118 & $T_{3} x^{6}$ & 1.53 & 0.38 & 90.33 & 5.84 & 1.23 & 0.17 & 0.19 & 0.07 & 0.03 & 0.16 & 98.01 & 0.87 & 2.41 & 0.922 \\
\hline & Guang'an 122 & $T_{3} x^{6}$ & 0.71 & 0.31 & 90.14 & 6.14 & 1.53 & 0.34 & 0.30 & 0.13 & 0.07 & 0.23 & 98.88 & 1.11 & 1.89 & 0.912 \\
\hline & Guang'an 130 & $T_{3} x^{6}$ & 0.00 & 0.76 & 86.22 & 6.00 & 1.75 & 0.37 & 0.36 & 0.17 & 0.09 & 0.24 & 95.20 & 1.03 & 2.01 & 0.906 \\
\hline & Guang'an 133 & $T_{3} x^{6}$ & 0.13 & 0.45 & 86.21 & 7.15 & 2.70 & 0.71 & 0.76 & 0.38 & 0.20 & 0.65 & 98.76 & 0.93 & 1.87 & 0.873 \\
\hline & Guang'an 15 & $\mathrm{~T}_{3} \mathrm{x}^{6}$ & 1.48 & 0.05 & 86.47 & 7.29 & 2.81 & 0.55 & 0.61 & 0.20 & 0.15 & 0.35 & 98.43 & 0.90 & 1.33 & 0.878 \\
\hline & Guang'an 2 & $T_{3} x^{6}$ & 0.15 & 0.19 & 89.03 & 6.65 & 1.93 & 0.39 & 0.40 & 0.16 & 0.09 & 0.34 & 98.99 & 0.96 & 1.68 & 0.899 \\
\hline & Guang'an 3 & $\mathrm{~T}_{3} \mathrm{x}^{6}$ & 0.97 & 0.20 & 90.92 & 4.20 & 1.74 & 0.26 & 0.53 & 0.18 & 0.21 & 0.70 & 98.74 & 0.49 & 0.86 & 0.921 \\
\hline & Guang'an 7 & $T_{3} x^{6}$ & 0.25 & 0.63 & 88.30 & 7.32 & 2.10 & 0.39 & 0.40 & 0.17 & 0.08 & 0.33 & 99.10 & 0.96 & 2.04 & 0.891 \\
\hline
\end{tabular}

\subsection{Characteristics of natural gas composition in Xujiahe Formation in the Central Sichuan basin}

The natural gas from Xujiahe Formation in the central Sichuan basin is dominantly composed of hydrocarbon gases, ranging between $95.2 \%$ and $99.5 \%$ with an average of $98.3 \%$. There is also minor 
amount of non-hydrocarbon gases such as $\mathrm{N}_{2}$ and $\mathrm{CO}_{2}$, with the average composition of $0.70 \%$ and $0.39 \%$, respectively. Among the hydrocarbon gases, heavy hydrocarbon gases such as ethane have high concentrations, and the dryness coefficient of natural gas $\left(\mathrm{C}_{1} / \mathrm{C}_{1+}\right)$ is between 0.803 and 0.972 , with an average of 0.910 . If the dryness coefficient of 0.95 is used as the boundary between dry gas and wet gas, the natural gas from Xujiahe Formation in the Central Sichuan basin is mainly wet gas (Table 1). $\mathrm{iC}_{4} / \mathrm{nC}_{4}$ and $\mathrm{iC}_{5} / \mathrm{nC}_{5}$ ratios are also significantly different within different sections of the Xujiahe Formation. In general, the lower the section, the higher the ratios. In $\mathrm{T}_{3} \mathrm{x}^{6}, \mathrm{~T}_{3} \mathrm{x}^{4}$ and $\mathrm{T}_{3} \mathrm{x}^{2}, \mathrm{iC}_{4} / \mathrm{nC}_{4}$ are $0.95,1.28$ and 1.29 respectively, and the ratios of $\mathrm{iC}_{5} / \mathrm{nC}_{5}$ are $1.87,2.20$ and 2.25, respectively. Different gas fields also have different ratios. For example, in the $\mathrm{T}_{3} \mathrm{x}^{2}$ gas reservoir, the value of $\mathrm{iC}_{4} / \mathrm{nC}_{4}$ reaches the highest in Hechuan gas field with an average of 1.46. However, the ratio drops down to the lowest value of 1.07 in Tongnan gas field.

4.2 The carbon isotopes in natural gases from the Xujiahe Formation in the central Sichuan basin is relatively more negative.

Carbon isotopes in alkane gases derived from coal (humic kerogen) is significantly less negative than the carbon isotopes in alkane gases derived from oil (sapropel kerogen), even they are generated from source rocks with similar maturity. Although alkane gases from the Xujiahe Formation in the Sichuan Basin belong to heavy carbon isotope series, the carbon isotope values in different regions are significantly different from each other. The carbon isotopes of alkane gases in the central Sichuan basin are relatively lighter than those in the alkane gases in the western Sichuan (Table 2, Figure 2). The carbon isotope of methane is remarkably different in gases from the central and western Sichuan basin. In gases from the central Sichuan basin, $\delta^{13} \mathrm{C}_{1}$ ranges from $-44.1 \%$ to $-37.1 \%$ with an average of $-40.1 \%$. While in the gases from the western Sichuan basin, $\delta^{13} \mathrm{C}_{1}$ is between $-35.5 \%$ and $-30 \%$ with an average of $-32.2 \%$. Difference of maturities between the Xujiahe formation in the central Sichuan basin and western Sichuan basin can explain the difference between methane carbon isotopic values in these two areas. The sedimentary center of Xujiahe Formation is located in the western Sichuan basin, which has a higher maturity level than that in the central Sichuan basin. This is consistent with the geological background. In the central and western Sichuan basin, there is a small difference in the carbon isotopes of ethane with the $\delta^{13} \mathrm{C}_{2}$ in the central part of Sichuan basin slightly lighter than that in the western Sichuan basin. In the central Sichuan basin, $\delta^{13} \mathrm{C}_{2}$ ranges between $-28.3 \%$ and $-25.9 \%$ with an average of $-27.5 \%$, and in the western Sichuan basin, $\delta^{13} \mathrm{C}_{2}$ is between $-28.1 \%$ and $-21.7 \%$ with an average of $-24.4 \%$. Although the carbon isotopes of methane are greatly affected by the maturity of source rocks, carbon isotopes in ethane and other heavy hydrocarbon have less variation with slightly heavier isotopes with the increase of maturity. Due to this fact, carbon isotopes of ethane have been used as the most important indicator to identify natural gas genetic types. 


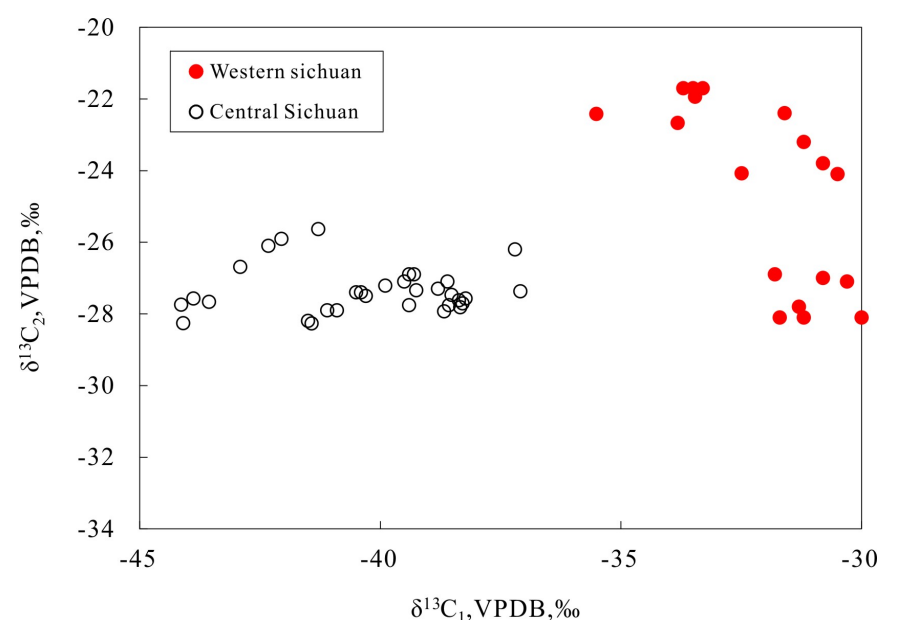

Fig. 2. $\delta^{13} C_{1}$ vs. $\delta^{13} C_{2}$ in natural gases from Xujiahe Formation in the Sichuan Basin

Table 2

Abundance and stable carbon isotopic values in natural gases from the Western and Central Sichuan Basin

\begin{tabular}{|c|c|c|c|c|c|c|c|c|c|c|c|c|c|c|c|c|}
\hline \multirow{2}{*}{ Gas field } & \multirow{2}{*}{ Well } & \multirow{2}{*}{ Strata } & \multicolumn{9}{|c|}{ Main molecular composition (\%) } & \multicolumn{4}{|c|}{$\delta^{13} \mathrm{C}, \mathrm{VPDB}(\%)$} & \multirow{2}{*}{ References } \\
\hline & & & $\mathrm{N}_{2}$ & $\mathrm{CO}_{2}$ & $\mathrm{C}_{1}$ & $\mathrm{C}_{2}$ & $\mathrm{C}_{3}$ & $\mathrm{iC}_{4}$ & $\mathrm{nC}_{4}$ & $\mathrm{iC}_{5}$ & $\mathrm{nC}_{5}$ & $\mathrm{C}_{1}$ & $\mathrm{C}_{2}$ & $\mathrm{C}_{3}$ & $\mathrm{C}_{4}$ & \\
\hline \multirow[t]{6}{*}{ Anyue } & Yue101-11 & $\mathrm{T}_{3} \mathrm{x}^{2}$ & 0.63 & 0.76 & 88.47 & 7.31 & 1.80 & 0.34 & 0.28 & 0.20 & 0.06 & -42.9 & -26.7 & -23.2 & -24.1 & This paper \\
\hline & Yue 103 & $\mathrm{~T}_{3} \mathrm{x}^{2}$ & 0.47 & 0.33 & 89.15 & 7.11 & 1.80 & 0.45 & 0.38 & 0.17 & 0.04 & -42.1 & -25.9 & -23.0 & -23.6 & \\
\hline & Yue 104 & $\mathrm{~T}_{3} \mathrm{x}^{2}$ & 0.13 & 3.05 & 85.24 & 8.25 & 2.46 & 0.39 & 0.33 & 0.10 & 0.03 & -43.6 & -27.7 & -24.7 & -25.5 & \\
\hline & Yue 114 & $\mathrm{~T}_{3} \mathrm{x}^{2}$ & 0.57 & 0.00 & 87.79 & 7.82 & 2.54 & 0.48 & 0.47 & 0.19 & 0.00 & -44.1 & -28.3 & -25.1 & -26.8 & \\
\hline & Yue 118 & $\mathrm{~T}_{3} \mathrm{x}^{2}$ & 0.61 & 0.29 & 87.89 & 7.85 & 2.31 & 0.38 & 0.37 & 0.12 & 0.03 & -43.9 & -27.6 & -24.7 & -25.6 & \\
\hline & Yue 137 & $\mathrm{~T}_{3} \mathrm{x}^{2}$ & 0.59 & 2.36 & 85.22 & 8.24 & 2.52 & 0.42 & 0.41 & 0.12 & 0.00 & -44.1 & -27.7 & -24.6 & -25.7 & \\
\hline \multirow[t]{5}{*}{ Tongnan } & Tongnan 101 & $T_{3} x^{2}$ & 0.93 & 0.16 & 87.27 & 7.26 & 2.74 & 0.53 & 0.53 & 0.23 & 0.11 & -41.5 & -28.2 & -25 & -26.1 & Qin et al.,2018 \\
\hline & Yue 111 & $T_{3} x^{2}$ & 0.72 & 0.17 & 87.95 & 7.12 & 2.48 & 0.53 & 0.48 & 0.23 & 0.10 & -41.1 & -27.9 & -24.7 & -25.6 & \\
\hline & Yue 104 & $\mathrm{~T}_{3} \mathrm{x}^{2}$ & 0.70 & 0.16 & 87.88 & 7.14 & 2.52 & 0.56 & 0.50 & 0.22 & 0.10 & -40.9 & -27.9 & -24.6 & -25.7 & \\
\hline & Yue 001-2 & $\mathrm{T}_{3} \mathrm{x}^{2}$ & 0.65 & 0.19 & 88.74 & 6.78 & 2.12 & 0.51 & 0.43 & 0.23 & 0.10 & -40.5 & -27.4 & -24.4 & -25.6 & \\
\hline & Yue 105 & $\mathrm{~T}_{3} \mathrm{x}^{2}$ & 0.60 & 0.14 & 89.57 & 6.49 & 1.85 & 0.43 & 0.35 & 0.19 & 0.08 & -40.3 & -27.5 & -24.5 & -25.0 & \\
\hline \multirow[t]{7}{*}{ Hechuan } & Hechuan 124 & $\mathrm{~T}_{3} \mathrm{x}^{2}$ & 0.78 & 0.15 & 89.19 & 6.57 & 1.68 & 0.44 & 0.33 & 0.21 & 0.09 & -40.4 & -27.4 & -24.5 & -25.3 & \\
\hline & Hechuan 106 & $\mathrm{~T}_{3} \mathrm{x}^{2}$ & 0.53 & 0.36 & 89.27 & 6.75 & 1.73 & 0.40 & 0.31 & 0.17 & 0.07 & -39.4 & -26.9 & -24.1 & -24.6 & \\
\hline & Hechuan 001-30-X1 & $\mathrm{T}_{3} \mathrm{X}^{2}$ & 0.87 & 0.22 & 90.31 & 6.07 & 1.36 & 0.36 & 0.26 & 0.16 & 0.06 & -39.5 & -27.1 & -24.3 & -24.8 & \\
\hline & Hechuan 108 & $\mathrm{~T}_{3} \mathrm{x}^{2}$ & 0.77 & 0.43 & 89.80 & 6.42 & 1.43 & 0.34 & 0.27 & 0.16 & 0.07 & -38.6 & -27.1 & -25.2 & -26.3 & \\
\hline & Hechuan 125 & $\mathrm{~T}_{3} \mathrm{x}^{2}$ & 0.69 & 0.12 & 92.83 & 4.82 & 0.82 & 0.23 & 0.15 & 0.11 & 0.04 & -37.2 & -26.2 & -24.5 & -26.3 & \\
\hline & Hechuan 001-18-X2 & $\mathrm{T}_{3} \mathrm{x}^{2}$ & 0.68 & 0.15 & 89.92 & 6.67 & 1.57 & 0.29 & 0.25 & 0.12 & 0.05 & -38.8 & -27.3 & -24.6 & & \\
\hline & Hechuan 001-2 & $\mathrm{T}_{3} \mathrm{x}^{2}$ & 0.78 & 0.18 & 89.53 & 6.71 & 1.68 & 0.39 & 0.28 & 0.15 & 0.06 & -39.3 & -26.9 & -23.9 & -24.0 & \\
\hline \multirow[t]{2}{*}{ Nanchong } & Xi 20 & $\mathrm{~T}_{3} \mathrm{x}^{4}$ & 0.64 & 0.36 & 88.68 & 6.07 & 2.41 & 0.48 & 0.52 & 0.21 & 0.12 & -41.4 & -28.3 & -25.3 & -24.4 & \\
\hline & Xi 51 & $\mathrm{~T}_{3} \mathrm{x}^{4}$ & 0.63 & 0.23 & 87.71 & 5.85 & 1.92 & 0.38 & 0.45 & 0.29 & 0.21 & -39.9 & -27.2 & -24.5 & -24.0 & \\
\hline \multirow[t]{10}{*}{ Guang'an } & Guang'an 002-11-H2 & $\mathrm{T}_{3} \mathrm{x}^{6}$ & 1.19 & 0.35 & 89.31 & 6.15 & 1.85 & 0.34 & 0.33 & 0.13 & 0.07 & -38.3 & -27.7 & -26.3 & -25.1 & \\
\hline & Guang'an 002-23 & $\mathrm{T}_{3} \mathrm{x}^{6}$ & 0.40 & 0.37 & 89.88 & 6.32 & 1.88 & 0.34 & 0.33 & 0.13 & 0.07 & -38.5 & -27.5 & -26.1 & -25.3 & \\
\hline & Guang'an 002-40 & $\mathrm{T}_{3} \mathrm{x}^{6}$ & 0.55 & 0.30 & 89.42 & 6.40 & 1.98 & 0.38 & 0.38 & 0.16 & 0.09 & -38.7 & -27.9 & -26.4 & -25.8 & \\
\hline & Guang'an 002-X37 & $\mathrm{T}_{3} \mathrm{x}^{6}$ & 0.82 & 0.59 & 89.01 & 6.33 & 2.01 & 0.38 & 0.38 & 0.15 & 0.08 & -38.3 & -27.8 & -26.0 & -25.4 & \\
\hline & Guang'an 002-X38 & $\mathrm{T}_{3} \mathrm{x}^{6}$ & 0.53 & 0.34 & 89.18 & 6.57 & 2.07 & 0.39 & 0.38 & 0.15 & 0.08 & -39.4 & -27.8 & -26.0 & -25.0 & \\
\hline & Guang'an 002-X70 & $\mathrm{T}_{3} \mathrm{x}^{6}$ & 0.61 & 0.39 & 88.76 & 6.63 & 2.23 & 0.42 & 0.41 & 0.16 & 0.09 & -38.6 & -27.8 & -26.3 & -25.4 & \\
\hline & Guang'an 002-X72 & $\mathrm{T}_{3} \mathrm{x}^{6}$ & 0.50 & 0.49 & 88.94 & 6.30 & 1.91 & 0.37 & 0.37 & 0.17 & 0.10 & -38.2 & -27.6 & -26.3 & -25.4 & \\
\hline & Guang'an 51 & $\mathrm{~T}_{3} \mathrm{x}^{6}$ & 0.70 & 0.59 & 89.58 & 6.22 & 1.80 & 0.33 & 0.31 & 0.13 & 0.07 & -38.4 & -27.6 & -26.0 & -25.3 & \\
\hline & Guang'an 1 & $\mathrm{~T}_{3} \mathrm{x}^{6}$ & 0.04 & 0.13 & 90.14 & 6.66 & 1.87 & 0.36 & 0.33 & 0.14 & 0.07 & -39.3 & -27.3 & -25.1 & -23.9 & Li et al, 2007 \\
\hline & Guang'an 11 & $\mathrm{~T}_{3} \mathrm{x}^{6}$ & 0.95 & 0.16 & 95.85 & 2.03 & 0.44 & 0.10 & 0.08 & 0.04 & 0.02 & -37.1 & -27.4 & -22.7 & -23.7 & Li et al, 2007 \\
\hline \multirow[t]{7}{*}{ Xinchang } & $\times 2$ & $\mathrm{~T}_{3} \mathrm{X}^{2}$ & 0.00 & 0.00 & 97.37 & 0.91 & 0.08 & 0.01 & 0.01 & 0.01 & 0.00 & -31.3 & -27.8 & -28.0 & & Leng et al, 2011 \\
\hline & $\times 3$ & $\mathrm{~T}_{3} \mathrm{X}^{2}$ & 0.00 & 0.00 & 97.31 & 0.96 & 0.09 & 0.01 & 0.01 & 0.00 & 0.00 & -31.2 & -28.1 & -25.1 & -23.8 & \\
\hline & X 851 & $\mathrm{~T}_{3} \mathrm{X}^{2}$ & 0.00 & 0.00 & 97.37 & 0.83 & 0.09 & 0.01 & 0.01 & 0.00 & 0.00 & -30.3 & -27.1 & & & \\
\hline & X 856 & $\mathrm{~T}_{3} \mathrm{X}^{2}$ & 0.00 & 0.00 & 97.19 & 0.86 & 0.07 & 0.01 & 0.01 & 0.00 & 0.00 & -30.8 & -27.0 & -26.5 & & \\
\hline & X 150 & $\mathrm{~T}_{3} \mathrm{X}^{2}$ & 0.00 & 0.00 & 96.95 & 0.74 & 0.08 & 0.01 & 0.01 & 0.00 & 0.00 & -30.0 & -28.1 & -27.3 & -22.7 & \\
\hline & X 202 & $\mathrm{~T}_{3} \mathrm{X}^{2}$ & 0.31 & 0.89 & 96.69 & 0.77 & 0.07 & 0.01 & 0.01 & 0.00 & 0.00 & -31.7 & -28.1 & & & \\
\hline & X 853 & $\mathrm{~T}_{3} \mathrm{X}^{2}$ & 0.00 & 0.00 & 97.07 & 0.79 & 0.08 & 0.01 & 0.01 & 0.00 & 0.00 & -31.8 & -26.9 & -25.7 & & \\
\hline \multirow[t]{4}{*}{ Pingluoba* } & Pingluo 1 & $\mathrm{~T}_{3} \mathrm{X}^{2}$ & 0.29 & 0.67 & 96.77 & 1.93 & 0.23 & 0.03 & 0.05 & 0.01 & 0.01 & -33.8 & -22.7 & -22.8 & & Qin et al., 2007 \\
\hline & Pingluo 9 & $\mathrm{~T}_{3} \mathrm{X}^{4}$ & 0.24 & 0.38 & 96.32 & 2.51 & 0.41 & 0.03 & 0.07 & 0.01 & 0.01 & -35.5 & -22.4 & -21.7 & -23.9 & \\
\hline & Pingluo 12 & $\mathrm{~T}_{3} \mathrm{X}^{2}$ & 0.22 & 0.65 & 96.87 & 1.81 & 0.33 & 0.03 & 0.05 & 0.01 & 0.01 & -33.5 & -21.9 & -21.1 & & \\
\hline & Pingluo 8 & $\mathrm{~T}_{3} \mathrm{X}^{2}$ & 0.32 & 0.68 & 96.50 & 2.13 & 0.25 & 0.03 & 0.05 & 0.01 & 0.01 & -32.5 & -24.1 & -19.4 & & \\
\hline
\end{tabular}




\begin{tabular}{|c|c|c|c|c|c|c|c|c|c|c|c|c|c|c|c|c|}
\hline & Pingluo 3 & $\mathrm{~T}_{3} \mathrm{x}$ & 0.54 & 0.76 & 97.14 & 1.98 & 0.24 & 0.08 & 0.02 & & & -33.3 & -21.7 & -21.2 & -20.3 & Fan et al., 2005 \\
\hline & Pingluo 6 & $\mathrm{~T}_{3} \mathrm{x}$ & 0.37 & 0.77 & 96.81 & 2.37 & 0.31 & 0.11 & 0.02 & & & -33.5 & -21.7 & -22.6 & -22.1 & \\
\hline & Pingluo 10 & $\mathrm{~T}_{3} \mathrm{x}$ & 0.39 & 0.81 & 96.78 & 2.34 & 0.33 & 0.13 & 0.02 & & & -33.7 & -21.7 & -22.7 & -22.5 & \\
\hline \multirow[t]{7}{*}{ Qiongxi } & QX 3 & $\mathrm{~T}_{3} \mathrm{x}$ & 0.25 & 1.67 & 93.30 & 3.91 & 0.63 & 0.10 & 0.08 & 0.01 & 0.01 & -33.1 & -23.0 & -2.7 & -20.3 & Dai et al., 2012b \\
\hline & QX 4 & $T_{3 x}$ & 0.24 & 1.47 & 93.52 & 3.91 & 0.62 & 0.10 & 0.08 & 0.01 & 0.01 & -32.9 & -23.2 & -23.0 & -22.0 & \\
\hline & QX 13 & $T_{3 x}$ & 0.25 & 1.47 & 93.30 & 3.91 & 0.63 & 0.10 & 0.08 & 0.01 & 0.01 & -33.1 & -23.0 & -22.7 & -20.3 & \\
\hline & QX 006-X1 & $\mathrm{T}_{3} \mathrm{x}^{2}$ & 0.26 & 1.36 & 93.17 & 4.12 & 0.71 & 0.13 & 0.11 & & & -31.6 & -22.4 & -22.4 & & Wu et al., 2011 \\
\hline & QX 6 & $\mathrm{~T}_{3} \mathrm{x}^{2}$ & 0.21 & 0.92 & 95.95 & 2.48 & 0.30 & 0.04 & 0.04 & & & -31.2 & -23.2 & -23.1 & -20.9 & \\
\hline & QX 14 & $\mathrm{~T}_{3} \mathrm{x}^{2}$ & 0.23 & 1.55 & 96.50 & 1.57 & 0.12 & 0.02 & 0.01 & & & -30.5 & -24.1 & -23.8 & & \\
\hline & QX 16 & $\mathrm{~T}_{3} \mathrm{x}^{2}$ & 0.23 & 1.39 & 96.46 & 1.74 & 0.16 & 0.02 & 0.02 & & & -30.8 & -23.8 & & & \\
\hline \multirow[t]{3}{*}{ Gongshanmiao } & Gong 16 & $\mathrm{~J}_{2} \mathrm{~S}$ & & & & & & & & & & -42.9 & -33.2 & -30.3 & -29.6 & Chen et al., 2005 \\
\hline & Gong 13 & $\mathrm{~J}_{1} 1$ & & & & & & & & & & -42.3 & -31.3 & -30.2 & -29.6 & \\
\hline & Gong 35 & $\mathrm{~J}_{1} \mathrm{dn}$ & & & & & & & & & & -48.5 & -35.3 & -31.0 & -29.8 & \\
\hline \multirow[t]{2}{*}{ Lianchi } & Lian 14 & $\mathrm{~J}_{1} 1$ & & & & & & & & & & -43.2 & -30.5 & -27.6 & -27.2 & \\
\hline & Lian 63 & $\mathrm{~J}_{1 \mathrm{Z}}$ & 0.00 & 0.21 & 82.34 & 9.68 & 5.02 & & & & & -45.4 & -34.2 & -30.6 & -29.6 & \\
\hline Jinhua & Jin 1 & $\mathrm{~J}_{1 \mathrm{Z}}$ & & & & & & & & & & -41.4 & -32.0 & -30.0 & -30.0 & \\
\hline Nanchong & Xi 021-x1 & $\mathrm{J}_{1} 1$ & 1.25 & 0.22 & 87.62 & 6.59 & 2.81 & 0.37 & 0.59 & 0.13 & 0.15 & -43.3 & -31.1 & -28.5 & -27.9 & This paper \\
\hline \multirow[t]{6}{*}{ Longgang } & LG 2 & $\mathrm{~J}_{1 \mathrm{Z}}$ & 0.94 & 0.46 & 77.71 & 12.24 & 4.46 & 0.63 & 1.38 & 0.63 & 0.65 & -47.6 & -33.0 & -28.1 & -27.4 & \\
\hline & LG 7 & $\mathrm{~J}_{1 \mathrm{Z}}$ & 0.54 & 0.95 & 74.42 & 12.82 & 6.01 & 0.89 & 1.76 & 0.61 & 0.61 & -47.5 & -32.3 & -27.7 & -26.8 & \\
\hline & LG 42 & $\mathrm{~J}_{1} 1$ & 1.17 & 0.09 & 66.57 & 16.02 & 9.79 & 1.51 & 2.82 & 0.61 & 0.67 & -46.0 & -33.2 & -28.7 & -28.1 & \\
\hline & LG 18 & $\mathrm{~J}_{2} \mathrm{~S}$ & 63.62 & 0.12 & 33.78 & 1.08 & 0.36 & 0.05 & 0.11 & 0.04 & 0.17 & -43.5 & -36.8 & -30.0 & -27.6 & \\
\hline & LG 18 & $\mathrm{~T}_{2} \mathrm{l}$ & 0.12 & 4.59 & 94.34 & 0.79 & 0.07 & 0.01 & 0.01 & & & -36.5 & -35.5 & -30.5 & -27.1 & \\
\hline & LG 176 & $\mathrm{~T}_{2} \mathrm{l}$ & 0.34 & 2.42 & 95.16 & 1.71 & 0.23 & 0.02 & 0.02 & & & -37.8 & -32.5 & -30.6 & & \\
\hline \multirow[t]{8}{*}{ Moxi } & Mo 004-H9 & $\mathrm{T}_{2} \mathrm{l}$ & 0.59 & 0.00 & 99.12 & 0.16 & 0.00 & 0.00 & 0.00 & 0.00 & 0.00 & -35.0 & -32.8 & & & \\
\hline & Mo 140 & $\mathrm{~T}_{2} \mathrm{l}$ & 0.23 & 0.00 & 99.54 & 0.17 & 0.00 & 0.00 & 0.00 & 0.00 & 0.00 & -35.0 & -32.4 & & & \\
\hline & Mo 144 & $\mathrm{~T}_{2} \mathrm{l}$ & 0.75 & 0.00 & 98.90 & 0.18 & 0.00 & 0.00 & 0.00 & 0.00 & 0.00 & -34.9 & -32.1 & & & \\
\hline & Mo 005-H10 & $\mathrm{T}_{1 \mathrm{j}}$ & 0.44 & 0.00 & 99.24 & 0.21 & 0.00 & 0.00 & 0.00 & 0.00 & 0.00 & -34.6 & -34.6 & & & \\
\hline & Mo 005-H9 & $\mathrm{T}_{1 \mathrm{j}}$ & 0.81 & 0.00 & 98.81 & 0.19 & 0.00 & 0.00 & 0.00 & 0.00 & 0.00 & -34.8 & -33.6 & & & \\
\hline & Mo 150 & $\mathrm{~T}_{1 \mathrm{j}}$ & 0.21 & 0.04 & 99.50 & 0.21 & 0.00 & 0.00 & 0.00 & 0.00 & 0.00 & -34.7 & -33.7 & & & \\
\hline & Mo 160 & $\mathrm{~T}_{1 \mathrm{j}}$ & 1.29 & 0.18 & 98.29 & 0.24 & & & & & & -32.3 & -34.0 & & & \\
\hline & Mo 5 & $\mathrm{~T}_{1 \mathrm{j}}$ & 0.80 & 0.00 & 98.85 & 0.17 & 0.00 & 0.00 & 0.00 & 0.00 & 0.00 & -34.6 & -33.2 & & & \\
\hline
\end{tabular}

* Xinchang, Pingluoba and Qiongxi gas fields are located in the Western Sichuan Basin, other fields are located in the Central Sichuan Basin.

\section{Discussion}

5.1 Natural gas in the Xujiahe Formation in the central Sichuan basin is not migrated from the western Sichuan basin.

Since the thickness of the Xujiahe Formation and the thickness of the coal-based source rocks in the central Sichuan basin are much smaller than those in the western Sichuan basin, the gas generation intensity of the source rocks in the Xujiahe Formation in the central Sichuan Basin is much smaller than that in the western Sichuan basin. As shown in Fig. 1, the highest intensity of gas generation in the Xujiahe Formation in the Central Sichuan is only $20 \times 10^{8} \mathrm{~m}^{3} / \mathrm{km}^{3}$. Based on previous research and exploration experience, it is not likely that a large gas field can be formed at such a low intensity of source rock. However, the studies on the geochemical characteristics of natural gas show that the natural gas in the Xujiahe Formation in the central Sichuan basin is a typical coal-type gas. Therefore, some researchers suggested that the natural gas in the Xujiahe Formation in the central Sichuan basin might come from the coal source rocks of the Xujiahe Formation in the western Sichuan basin. In this model, the natural gas generated from coal source rocks in the Xujiahe Formation traveled long distance to the central Sichuan Basin and accumulated in the Xujiahe Formation reservoir. However, it is a challenge to explain how the migration of natural gas can happen in terms of migration channels. The Xujiahe Formation and the overlying Jurassic and the underlying Leikoupa Formation are all sealed by non-permeable mudstone and natural gas cannot migrate along the contact surfaces between different formations. In addition, the reservoirs in both Western and Central Xujiahe Formation are tight sandstones, it is unclear whether natural gas can migrate long distances in the dense layer and there is no reliable conclusion so far. According to the results in Table 1 and Table 2, the geochemical characteristics of natural gas in the western Sichuan basin is significantly different from 
the gases from the Xujiahe Formation in the Central Sichuan Basin, both of which belong to the natural gas generated from coal with different maturity levels. As discussed previously, first of all, the dryness coefficients are different in two areas. Gases in the Central Sichuan Basin are mainly dry gas, and on the contrary, gases in the Western Sichuan basin are mainly wet gas (Fig. 3). Secondly, the carbon isotopes of alkanes from the Xujiahe Formation in the Central Sichuan Basin is lighter than those from the western Sichuan basin. In a Bernard diagram (Fig. 4), the natural gases in the Central and Western Xujiahe Formation are also lain in different regions. Gases from the Xujiahe Formation in the central Sichuan basin are in the region of thermogenic gas while gases from the Western Xujiahe Formation are in the type III kerogen region.

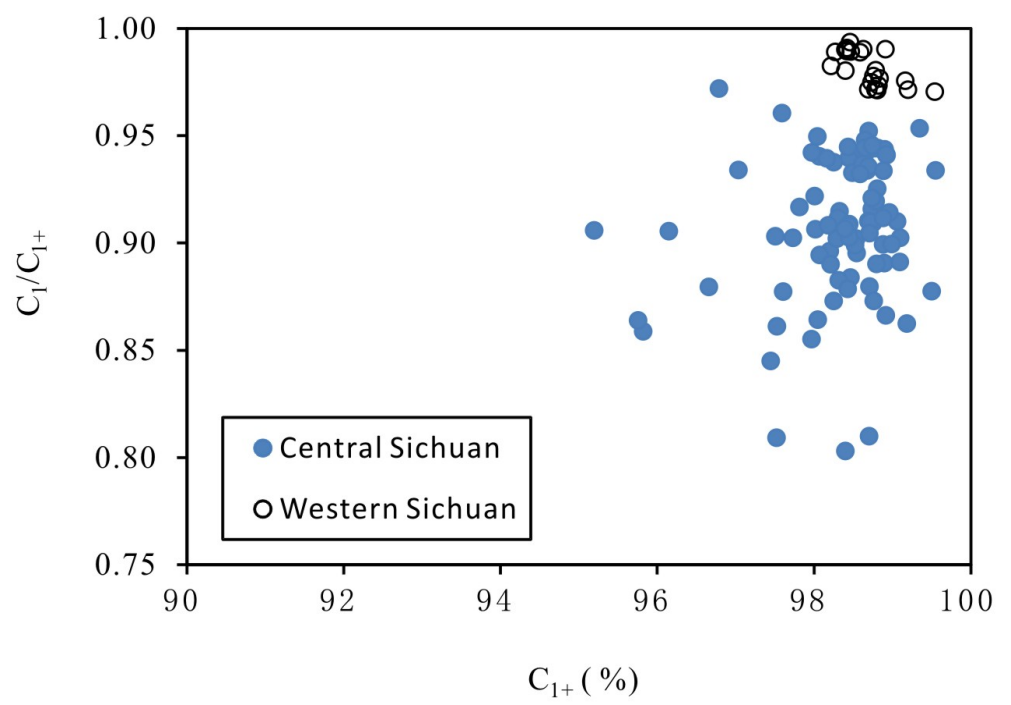

Fig.3. Relationship between natural gas dryness coefficient $\left(C_{1} / C_{1+}\right)$ and total hydrocarbon gas content in the Xujiahe Formation in the Sichuan Basin.

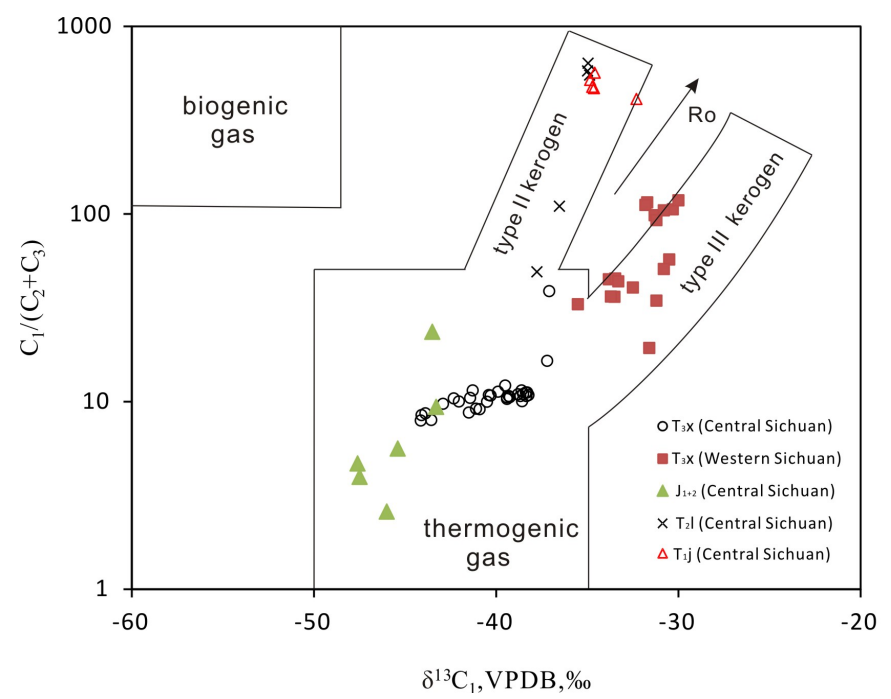

Fig.4. The 'gas wetness' $C_{1} /\left(C_{2}+C_{3}\right)$ vs. $\delta^{13} C_{1}$ showing the differences of gases listed in Table 2 (modified from Bernard et al. (1978) and Whiticar (1999).

In addition, if the natural gas from the Xujiahe Formation in the central Sichuan basin came from the western Sichuan basin, the fractionation effect of natural gas components would be very obvious over such a long distance, especially in tight formations. Along the migration pathway, concentrations of molecules with small diameters and weights should increase. Natural gas dryness coefficient should also increase gradually. As a consequence, dryness coefficient in the natural gas from Xujiahe Formation should be 
higher than that in the western Sichuan. But our data show opposite direction (Figure 3). Therefore, we propose that gases in the Xujiahe Formation in the Central Sichuan basin are derived from the source rocks within the Xujiahe Formation in the Central Sichuan basin. They are not from the western Sichuan basin.

Difference between gases from the Xujiahe Formation in the central Sichuan basin and those from the western Sichuan basin is consistent with the geological background in both areas. According to the measured Ro values of the source rocks, the maturity of the source rocks in the Xujiahe Formation in the central Sichuan basin is indeed lower than that in the western Sichuan basin (Dai et al., 2012b).

5.2 There is no horizontal migration of natural gases among adjacent gas fields in the Xujiahe Formation in the Central Sichuan basin

In previous section, distinct geochemical characteristics in gases from the Western and Central Sichuan basin suggested that long distance gas migration did not occur. A question still remains that if there is significant lateral migration of natural gases between adjacent gas fields in the central Sichuan basin. To test this hypothesis, we selected the Hechuan and Tongnan gas fields in the southern part of the central Sichuan basin and the Guang'an and Nanchong gas fields in the northern part of the central Sichuan basin for our study. Gases in these fields are all from the Xujiahe Formation.

The proven natural gas reserves in the Hechuan and Tongnan gas fields are accumulated in $T_{3} \mathrm{X}^{2}$ section. Although these two gas fields are not far from each other, the carbon isotopes of methane and ethane are significantly different. The natural gas in $\mathrm{T}_{3} \mathrm{X}^{2}$ gas reservoir in the Hechuan gas field is obviously heavier than that in the Tongnan gas field. The average carbon isotope ratios of methane and ethane in the Hechuan gas field are $-39.0 \%$ and $-27.0 \%$ respectively. The average carbon isotope ratios of methane and ethane in the Tongnan gas field are $-40.9 \%$ and $-27.8 \%$ respectively (Table 2, Figure 5). This suggests that the maturity of source rocks in the Hechuan gas field is higher than that in the Tongnan gas field. In addition, the natural gas dryness coefficient of the Hechuan Gas Field is significantly higher than that of the Tongnan Gas Field (Figure 6a). This is also due to the difference between the maturity of the source rocks and it is not caused by fractionation of gases due to lateral migration, because if the high dryness coefficient was due to fractionation associated with migration, the carbon isotope in methane in the Hechuan gas field should not be heavier than that in the Tongnan gas field as we observed.

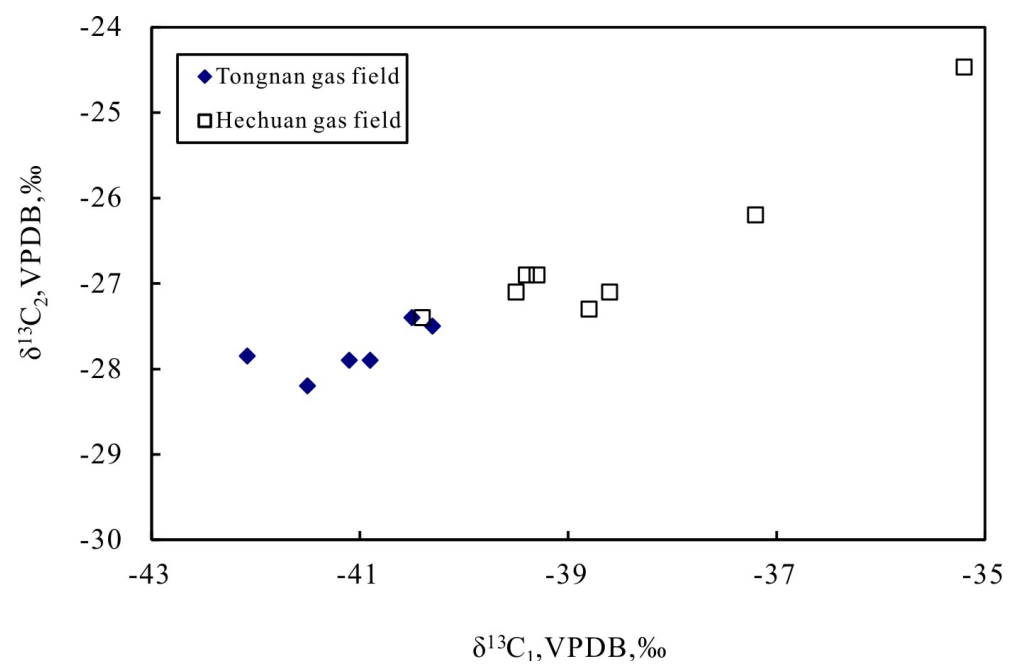

Fig. 5. $\delta^{13} C_{1}$ vs. $\delta^{13} C_{2}$ in natural gases from $T_{3} x^{2}$ reservoirs in the Hechuan and Tongnan gas fields.

Isomeric ratios in alkane gas in the Hechuan and Tongnan gas fields are also significantly different. They are higher in the Hechuan gas field than in the Tongnan gas field (Figure 6b). Due to low boiling point, high saturation vapor pressure and small intermolecular force, the diffusion coefficients of isomers of alkane gases are higher than normal alkane gases with the same carbon number. Therefore, $\mathrm{iC}_{4}$ and $\mathrm{iC}_{5}$ 
migrate faster than $\mathrm{nC}_{4}$ and $\mathrm{nC}_{5}$, respectively. Previous research shows that the diffusivity of $\mathrm{iC}_{4}$ is greater than that of $\mathrm{nC}_{4}$ in shale saturated with brine at $38{ }^{\circ} \mathrm{C}$, which are $1.26 \times 10^{-7} \mathrm{~cm}^{2} / \mathrm{s}$ and $1.24 \times 10^{-7} \mathrm{~cm} / \mathrm{s}$, respectively. The diffusivity of $\mathrm{iC}_{5}$ is larger than that of $\mathrm{nC}_{5}$, which are $7.0 \times 10^{-7} \mathrm{~cm}^{2} / \mathrm{s}$ and $5.2 \times 10^{-7} \mathrm{~cm}^{2} / \mathrm{s}$, respectively (Hao et al., 1994). When gas chromatography is used for determination of natural gas abundances, isoparaffins are detected earlier than normal alkanes with the same carbon number. As a result, the ratios of $\mathrm{iC}_{4} / \mathrm{nC}_{4}$ and $\mathrm{iC}_{5} / \mathrm{nC}_{5}$ increase with the increase of gas migration distance. In this sense, the difference between the isomeric ratio of alkane gases from the Tongnan and Hechuan gas fields may also be explained by migration of natural gases. However, this contradicts the fact that methane isotopes in the gases from the Hechuan field are heavier than those from the Tongnan field. In general, natural gas tends to migrate from source rocks with high maturity to source rocks with low maturity. Therefore, under the same geological conditions, the gas generation intensity of source rock would be relatively high if the maturity of the source rock is high.
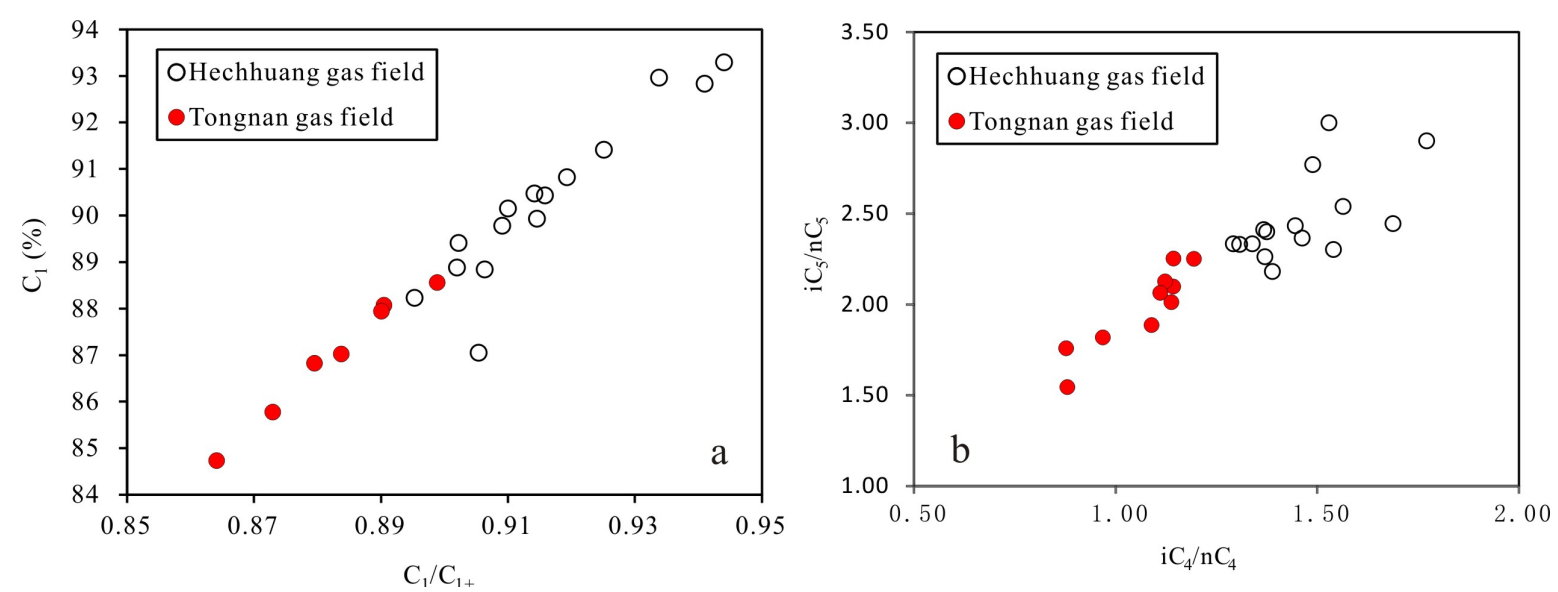

Fig. 6. Comparison of geochemical parameters in natural gases from the $T_{3} x^{2}$ reservoirs in the Hechuan and Tongnan gas fields

There is also an obvious difference in geochemical characteristics between gases from the Nanchong gas field and Guang'an gas field in the northern part of the central Sichuan basin. Althogh gases in both fields are from the $\mathrm{T}_{3} \mathrm{x}^{4}$ section of the Xujiahe Formation, the natural gas from the Guangxian field has a dryness coefficient larger than that in the Nanchong gas field. The isomerization of alkane gas is also obviously higher in the Guangxian field than that in the the Nanchong field. This difference can also be explained by the difference between the maturities of source rocks in both gas reservoirs.
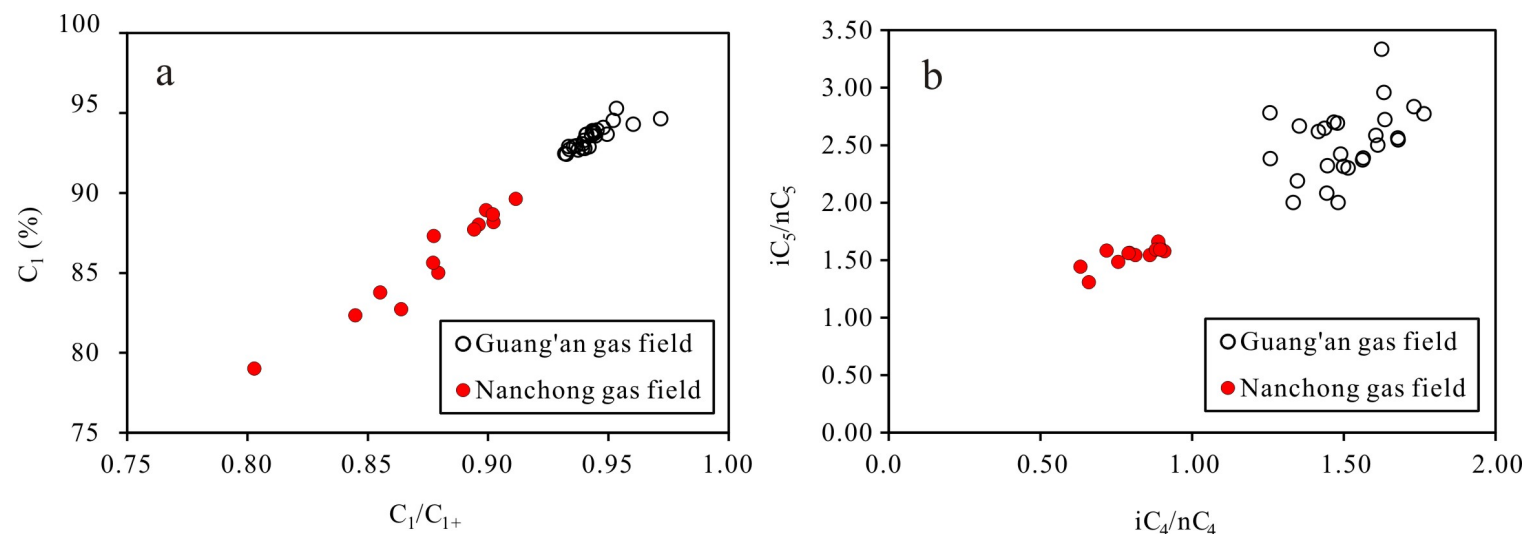

Fig. 7. Comparison of geochemical parameters in natural gases from the $T_{3} x^{4}$ reservoirs in the Guang'an and Nanchong gas fields 
Based on the evidence that geochemical characteristics of the adjacent Xujiahehe gas fields in the central Sichuan basin are significantly distinct from each other and the difference can be caused by the different maturities of the source rocks which supply gases in the respective reservoirs, there is no significant lateral migration of natural gas among the Xujiahe Formation gas reservoirs in horizontal direction.

5.3 Gas reservoirs in the Xujiahe Formation in the central Sichuan basin are not intermixed with natural gases from other adjacent formations vertically

The Xujiahe Formation in the Central Sichuan Basin is overlaid by the formation of Jurassic age. Within it, the Lower Jurassic formation is characterized by thick, organic-rich gray-black shale, which is composed of lacustrine sediments with low form of organisms (Du et al., 2005). This is different from the coal derived source rocks in the Xujiahe Formation. In the Middle and Lower Jurassic formation, both petroleum reservoirs and associated gases were found. The Xujiakou Formation and the Jialingjiang Formation were found below the Xujiahe Formation. Although both the Leikoupo Formation and the Jialingjiang Formation are marine strata, It has not been determined whether effective source rocks have been developed in these formations. However, gas reservoirs have been discovered in both formations. Whether gases derived from coal-based source rock in the Xujiahe Formation migrated into the overlying Jurassic and underlying Leikoupo and Jialingjiang formations and whether gases from these formations migrated into the Xujiahe Formation remain an open question.

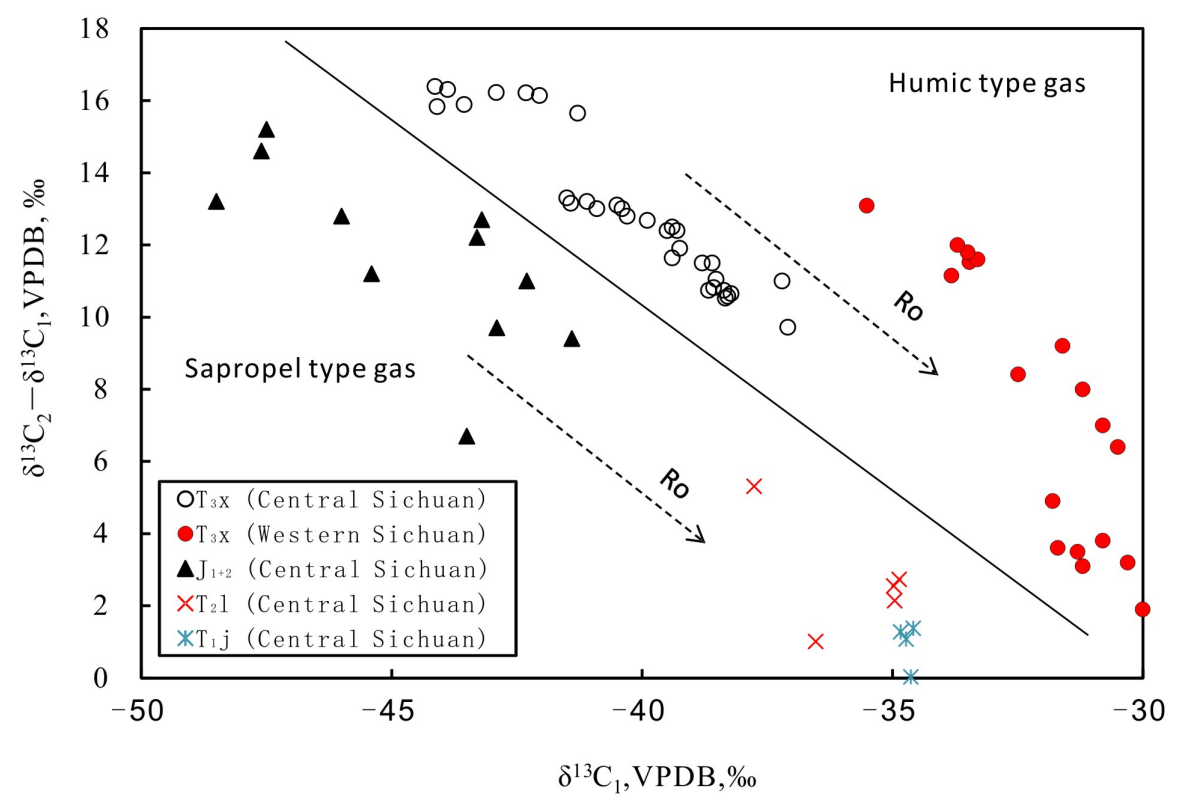

Fig. 8. Genetic natural gas types in the Central and Western Sichuan basin

It is shown in Table 2 and Figure 8 that the natural gases in the Xujiahe Formation in the Central Sichuan basin are clearly distributed in different regions with gases in the Jurassic, Leikoupo and Jialingjiang Formations being different gas types. The gas in the Xujiahe Formation is distributed in the coal-type gas region, and the natural gases in the Jurassic, Leikoupo and Jialingjiang formations are distributed in the oil-type gas region. Due to low maturity of Jurassic formation and lighter carbon isotopes in methane, natural gas in this formation is distributed in the region with low maturity. The natural gases in the Lekoukou and Jialingjiang formations are distributed in regions with high maturity. This geochemical characteristic of natural gas is consistent with the geological background. Source rocks in the Jurassic formation are shallow-deep lacustrine sediments. Organic matter is mainly sapropelic, which is type I-II kerogen. Both the Leikoupo and Jialingjiang formations and strata below are marine sediments with natural 
gas originated from marine source rocks. Organic matter in the marine source rocks is generally sapropelic, which is kerogen type I - II. Therefore, the natural gas in the Xujiahe Formation in the central Sichuan basin has neither migrated into the adjacent strata, nor has the natural gas from the adjacent strata migrated into the Xujiahe Formation.

5.4 There is distinct difference between gases from upper and lower gas reservoirs in the Xujiahe Formation in the Central Sichuan basin

By taking Guang'an gas field as an example, which produces gases from both the upper and lower Xujiahe formation, we aim to study the migration of gases within the Xiujiahe Formation. The main gas reservoirs in the Guang'an gas field are located in the $\mathrm{T}_{3} \mathrm{x}^{4}$ and $\mathrm{T}_{3} \mathrm{x}^{6}$ sections, with natural gas most likely coming from coal-based source rocks in the $\mathrm{T}_{3} \mathrm{x}^{3}$ and $\mathrm{T}_{3} \mathrm{x}^{5}$ sections below the gas reservoirs. Source rokcs in the $\mathrm{T}_{3} \mathrm{x}^{3}$ section is more mature than those in the $\mathrm{T}_{3} \mathrm{x}^{5}$ section.

If natural gas does migrate from the $T_{3} x^{4}$ gas reservoir to the $T_{3} x^{6}$ gas reservoir, the natural gas dryness coefficient, the alkane gas abundance and isomeric ratios in the $T_{3} \mathrm{x}^{6}$ gas reservoir should be higher than those in the $T_{3} x^{4}$ gas reservoir. However, results show opposite direction. As can be seen in Figure 9, the natural gas dryness coefficient and the isomeric ratio of alkane gases in the $T_{3} x^{4}$ gas reservoir are significantly higher than those in the $\mathrm{T}_{3} \mathrm{x}^{6}$ gas reservoir. This phenomenon can be reasonably explained by difference in the maturities of source rocks. The distinctive geochemical parameters of natural gas in the $\mathrm{T}_{3} \mathrm{x}^{4}$ and $\mathrm{T}_{3} \mathrm{X}^{6}$ gas reservoirs also illustrate that there is no major mixing of natural gas in the upper and lower gas reservoirs in the Xujiahe Formation in the Central Sichuan Basin.
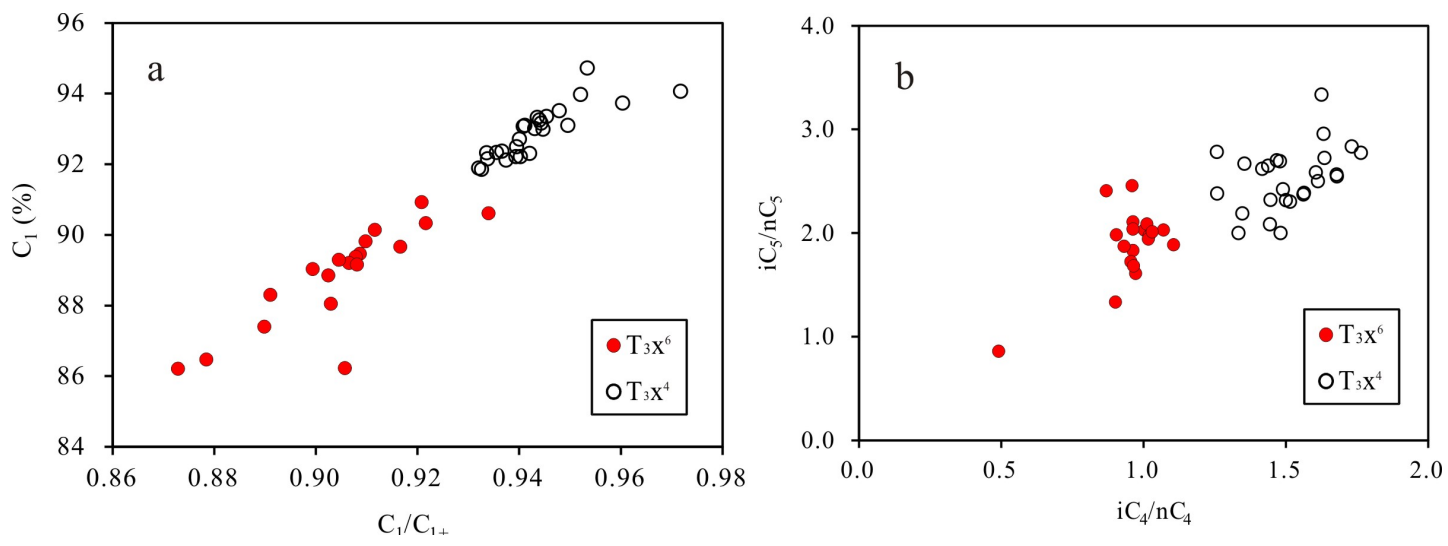

Fig. 9. Comparison of geochemical parameters in natural gases from $T_{3} x^{4}$ and $T_{3} x^{6}$ reservoirs in the Guang'an gas field.

We sampled a number of wells where gas samples can be taken from both the $T_{3} \mathrm{x}^{4}$ and $\mathrm{T}_{3} \mathrm{x}^{6}$ gas reservoirs individually to study difference between the upper and lower gas reservoirs. Gas wetness coefficient $\left(C_{1} /\left(C_{2}+C_{3}\right)\right)$ in the $T_{3} x^{4}$ gas reservoir is significantly higher than that in the $T_{3} x^{6}$ gas reservoir. Heavy hydrocarbon content in the $\mathrm{T}_{3} \mathrm{x}^{6}$ gas reservoir is higher than that in the $\mathrm{T}_{3} \mathrm{x}^{4}$ reservoir (Fig. 10).

We also listed density and methane content of natural gases in some wells drilled through the entire Xujiahe Formation in the Guang'an field and other fields to compare geochemical characteristics of natural gas at different depth of reservoirs (Table 3). Comparisons revealed that, from bottom to top, which is from $T_{3} x^{2}$ to $T_{3} x^{4}$ and $T_{3} x^{6}$ section, the natural gas density increased, and the methane content decreased (Table 3). This is consistent with the gradual decrease of maturity from $T_{3} x^{1}$ to $T_{3} x^{3}$ and $T_{3} x^{5}$ source rocks. It also demonstrates that there is no mixing of natural gas between the upper and lower gas reservoirs in the Xujiahe Formation. Natural gas in the Xujiahe Formation is accumulated in situ and has not undergone any major migration process after generation. 

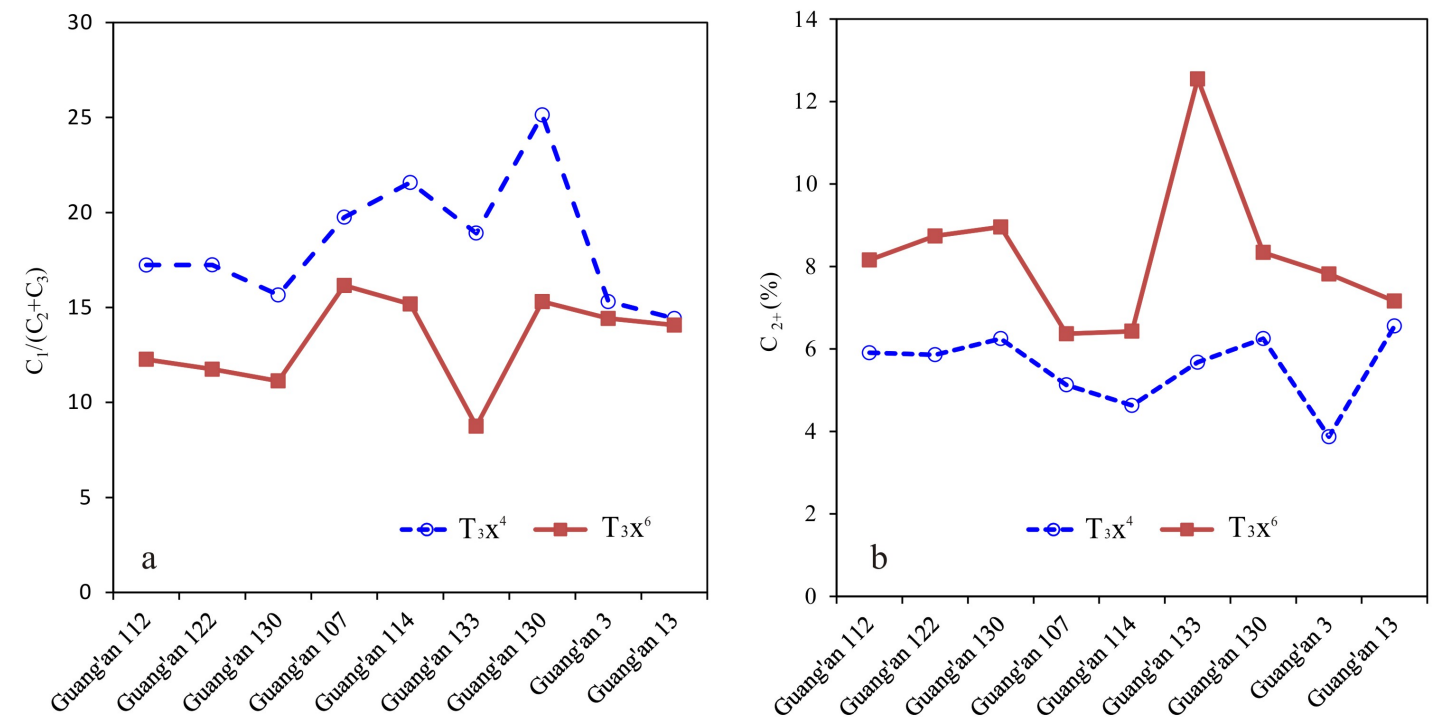

Fig. 10. Comparison of gas geochemical parameters in gases from $T_{3} x^{4}$ and $T_{3} x^{6}$ reservoirs in the same wells in the Guang'an gas field.

Table 3

Density and methane content of natural gases in the Xujiahe Formation in the Central Sichuan basin

\begin{tabular}{|c|c|c|c|c|c|c|c|}
\hline \multirow{2}{*}{ Gas field } & \multirow{2}{*}{ Well } & \multicolumn{2}{|c|}{$\mathrm{T}_{3} \mathrm{x}^{2}$} & \multicolumn{2}{|c|}{$\mathrm{T}_{3} \mathrm{x}^{4}$} & \multicolumn{2}{|c|}{$T_{3} x^{6}$} \\
\hline & & Density & $\mathrm{CH}_{4}(\%)$ & Density & $\mathrm{CH}_{4}(\%)$ & Density & $\mathrm{CH}_{4}(\%)$ \\
\hline Bajiaochang & Jiao 48 & 0.602 & 93.00 & 0.620 & 91.00 & 0.637 & 88.00 \\
\hline Suinan & Sui 12 & 0.638 & 89.00 & 0.648 & 86.00 & 0.686 & 83.00 \\
\hline Moxi & Mo 12 & 0.638 & 88.00 & 0.648 & 86.00 & & \\
\hline \multirow[t]{3}{*}{ Guang'an } & Guang'an 112 & & & 0.603 & 92.70 & 0.621 & 89.66 \\
\hline & Guang'an 114 & & & 0.587 & 94.72 & 0.614 & 90.72 \\
\hline & Guang'an 133 & & & 0.610 & 92.30 & 0.670 & 86.21 \\
\hline
\end{tabular}

5.5 In - situ accumulation of tight gas in the Xujiahe Formation in the central Sichuan basin is due to specific local geological setting

5.5.1 Development of favorable seals on both bottom and top of the Xujiahe Formation

First of all, the Xujiahe Formation, underlying Middle Triassic formations and overlying Jurassic formations in the Central Sichuan basin are sealed off by non-permeable rocks, which are not in favor of migration of natural gas in the Xujiahe Formation (Jiang et al., 2006). Secondly, the thick shale, developed at the bottom of the Lower Jurassic formation, not only provide oil and gas sources for the Jurassic reservoirs, but also provide high-quality caprock for Xujiahe Formation gas reservoirs. The Middle Triassic Leikoupo Formation and the Lower Triassic Jialingjiang Formation have all developed gypsum salt layers, which are good barriers to prevent the natural gas from migrating downwards.

5.5.2 Relatively stable tectonic history makes the in situ gas accumulate in the Xujiahe Formation possible

Tight sandstone reservoirs are widely distributed in the Xujiahe Formation. However, strong heterogeneity of the reservoirs and gentle slope of the formation limit long-distance migration of oil and gas. Due to stable regional tectonic activities, the regional tectonic stress in sedimentary formation is weak. As a consequence, faults and fissures are not developed. Therefore, hydrocarbon migration in tight sandstone is limited (Jiang et al., 2006). The regional overpressure of the fluids in the reservoir also reflects that fluid migration in the Xujiahe Formation in the Central Sichuan Basin is hindered. The reservoirs in the Xujiahe Formation in the central Sichuan basin are overpressured in general, and the overpressure in 
different regions at the same depth varies (Table 4). This suggests that the reservoirs in the Xujiahe Formation have low porosity and permeability, poor reservoir connectivity, strong heterogeneity. There is no effective "pressure release mechanism", resulting in overpressure anomaly and heterogeneous pressure distribution in the Xujiahe Formation in the Central Sichuan basin.

Table 4

Statistics of stratigraphic pressure in the Xujiahe Formation in the Central Sichuan basin

\begin{tabular}{|c|c|c|c|c|c|}
\hline Gas field & Well & Measured depth $(\mathrm{m})$ & Strata & Pressure (MPa) & Pressure coefficient \\
\hline \multirow[t]{2}{*}{ Moxi } & Mo 11 & 2146 & $T_{3} x^{2}$ & 30.84 & 1.47 \\
\hline & Mo 76 & 2072.2 & $\mathrm{~T}_{3} \mathrm{x}^{2}$ & 30.17 & 1.48 \\
\hline \multirow[t]{2}{*}{ Tongnan } & Tongnan 101 & 2241.4 & $\mathrm{~T}_{3} \mathrm{x}^{2}$ & 31.60 & 1.44 \\
\hline & Tongnan 102 & 2240.85 & $\mathrm{~T}_{3} \mathrm{x}^{2}$ & 29.65 & 1.35 \\
\hline \multirow[t]{3}{*}{ Hechuan } & Hechuan 1 & 2135 & $\mathrm{~T}_{3} \mathrm{x}^{2}$ & 22.57 & 1.08 \\
\hline & Hechuan 3 & 2130.8 & $T_{3} x^{2}$ & 25.28 & 1.21 \\
\hline & Hechuan 5 & 2265 & $\mathrm{~T}_{3} \mathrm{x}^{2}$ & 30.07 & 1.35 \\
\hline \multirow[t]{3}{*}{ Nachong } & Chongshen 1 & 2205.6 & $\mathrm{~T}_{3} \mathrm{x}^{4}$ & 31.23 & 1.44 \\
\hline & Chongshan 2 & 2225.25 & $\mathrm{~T}_{3} \mathrm{x}^{4}$ & 29.03 & 1.33 \\
\hline & Chongshen 1 & 2205.6 & $\mathrm{~T}_{3} \mathrm{x}^{4}$ & 31.23 & 1.44 \\
\hline \multirow[t]{2}{*}{ Bajiaochang } & Jiao 13 & & $\mathrm{~T}_{3} \mathrm{x}^{4}$ & 55.14 & 1.81 \\
\hline & Jiao 45 & & $T_{3} x^{4}$ & 56.19 & 1.79 \\
\hline \multirow[t]{6}{*}{ Guang'an } & Guang'an 135 & 2475.22 & $\mathrm{~T}_{3} \mathrm{x}^{4}$ & 36.19 & 1.49 \\
\hline & Guang'an 138 & 2526.75 & $\mathrm{~T}_{3} \mathrm{x}^{4}$ & 32.41 & 1.31 \\
\hline & Guang'an 139 & 2370.4 & $T_{3} x^{4}$ & 35.72 & 1.54 \\
\hline & Guang'an 2 & 1782.45 & $\mathrm{~T}_{3} \mathrm{x}^{6}$ & 19.55 & 1.12 \\
\hline & Guang'an 131 & 2589 & $T_{3} x^{6}$ & 34.41 & 1.36 \\
\hline & Guang'an 103 & 1799.7 & $T_{3} x^{6}$ & 18.38 & 1.04 \\
\hline
\end{tabular}

6. Conclusion

Although the Central Sichuan basin has experienced many tectonic movements, it has not experienced large-scale extrusion deformation. The structures in the central Sichuan basin is relatively stable. There is no effective communication system formed in the tight reservoirs in the Xujiahe Formation, which limits the migration of natural gas. Geochemical studies of natural gas show that the natural gas in the Xujiahe Formation is accumulated in independent reservoirs. There is no significant horizontal and vertical migration between the gas reservoirs in the Xujiahe Formation. The tight gas in the Xujiahe Formation is generated and accumulated in situ.

\section{Acknowledgements}

This study was sponsored by National Natural Science Foundation of China (Grant No. 41372150). We thank editor for handling this manuscript.

\section{Reference}

Bernard, B.B., Brooks, J.M., Sackett, W.M., 1978. Light hydrocarbons in recent Texas continental shelf and slope sediments. Journal of Geophysical Research 83, 4053-4061.

Bian, C., Wang, H., Wang, Z., Li, Y., Zhu, r., Liu, L., 2009.Controlling factors for massive accumulation of natural gas in the Xujiahe Formation in central Sichuan Basin. Oil \& Gas Geology 30(5), 548-565 (in Chinese).

Che, G., Gong, C., Wang, N., Song, H., Li, J., 2007. Gas accumulation condition in the Xujiahe Group of Guang'an area. 
Natural Gas Industry 27(6), 1-5 (in Chinese).

Chen, T., Jia, A., He, D., Shao, H., Ji, L., Yan. H., 2014. Mechanisms of gas-water distribution in tight sandstone gas reservoirs of Xujiahe Formation, central Sichuan Basin. Oil \& Gas Geology 35(2): 218-223 (in Chinese).

Dai, J., Wang, T., Song, Y., Zhang, H., Xu, Y., Zhang, Q., 1997. Formation and distribution of medium-large-sized gas field in China. Geological Publishing House, Beijing (in Chinese).

Dai, J., Ni, Y., Wu, X. 2012a. Tight gas in China and its significance in exploration and exploitation. Petroleum Exploration and Development 39(3), 158-166 (in Chinese).

Dai, J., Ni, Y., Zou C., 2012b. Stable carbon and hydrogen isotopes of natural gases source from the Xujiahe Formation in the Sichuan Basin, China. Organic geochemistry 43(1), 103-111.

Chen, Y., Jiang, Y., Guo, G., Yang, J., 2007. Simulation of the source rock thermal evolution history of Xiangxi Group of Upper Trassic in central Sichuan area. Journal of Southwest Petroleum University 29(2), 57-60 (in Chinese).

Du, M., Chen, S., Wan, M., Zheng, P., Li, D., 2005. Study on distribution and geochemical features Of Jurassic source rocks In Sichuan Basin. Natural Gas Exploration \& Development 28(2), 14-17, 69 (in Chinese)

Hao, S., Huang, yang Z., Yang, J., 1994. The Migration-Accumulation Equilibrium Theory and Application of Natural Gas. Beijing: Petroleum Industry Press, P12-13 (in Chinese).

Jia, C., Zheng, M., Zhang, Y., 2012. Unconventional hydrocarbon resources in China and the prospect of exploration and development. Petroleum Exploration and Development, 39(2), 129-136 (in Chinese)

Jiang, Y., Guo, G., Chen, Y., Li, Z., Lu, Z.,2006. Mechanism of gas reservoiring in Xujiahe Formation in central Sichuan Basin. Natural Gas Industry 26(11), 1-3 (in Chinese).

Li, D., Li, W., Wang, Z., Yang, J., 2007. Genetic type and source of gas in the Guang'an gasfield, central Sichuan[J]. Geology in China 34(5), 829-836 (in Chinese).

Liu, H., Xing, H., Jiang, Z., Wang, Y., 2005. Resource predication and exploration of Xujiahe Formation in center of Chuanxi foreland basin. Coal Geology \& Exploration 33(1), 25-27 (in Chinese).

Leng, J., Yang, K., Ye, J., Zhu, H., 2011. Carbon Isotope Features of Alkane Gases of Xujiahe Formation in Xiaoquan-Fenggu Structural Belt and Their Gas-source Correlation. Journal of Oil and gas Technology 33(6), 16-21 (in Chinese).

Fan, R., Zhou, H., Cai, K., 2005. Carbon isotopic Geochemistry and origin of natural gases in the southern part of the Western Sichuan Depression. Acta Geoscientica Sinica 26(2), 157-162 (in Chinese).

Qin, S., Tao, S., Tu, T., Wei, X., Song, M., 2007. Characteristics of natural gas geochemistry and accumulation in Western Sichuan Depression. Petroleum Exploration and Development, 34(1), 34-38 (in Chinese).

Qin, S., Li, F., Li, W., Zhou, Z., Zhou, G., 2018. Formation mechanism of tight coal-derived-gas reservoirs with medium-low abundance in Xujiahe Formation, central Sichuan Basin, China. Marine and Petroleum Geology 89(1), 144-154.

Tong, X., Guo, B., Li, J., Huang, F., 2012. Comparison study on accumulation \& distribution of tight sandstone gas between China and the United States and its significance. Engineering Sciences, 14(6), 9-15, 30 (in Chinese).

Wei, G., Zhang, F., Li, J., Yang, S., Huang, C., She, Y., Jv, X., Zhao, L., 2016. New progress of tight sand gas accumulation theory and favorable exploration zones in China. Natural Gas Geoscience 27(2), 199-210 (in Chinese).

Wei, X., Hu, A., Zhao, H., Kang, R., Shi, X., Liu, X., 2017. New geological understanding of tight sandstone gas. Lithologic Reservoirs 29(1): 11-20 (in Chinese).

Whiticar, M.J., 1999. Carbon and hydrogen isotope systematics of bacterial formation and oxidation of methane. Chemical Geology 161, 291-314.

Xiao, Z., Xie, Z., Li, Z., Ma, C., 2008. Isotopic characteristics of natural gas of Xujiahe Formation in southern and middle of Sichuan Basin. Geochimica 37(3), 245-250 (in Chinese).

Xu, Z., Song, L., Wu, X., Chen, C., 2009. Typical gas reservoir and main controlling factors of reservoir-forming of Upper Triassic Xujiahe Formation in central Sichuan Basin. Lithologic Reservoirs 21(2), 7-11 (in Chinese).

Xie, J., Li, G., Luo, F., 2009. Reservoir Characteristics of the Upper Triassic Xujiahe Formation in Sichuan Basin, China. Journal of Chengdu University of Technology ( Science \& Technology Edition) 36(1),13-18.

Yang, Y., Wang, S., Huang, L., Zhong, J., 2009. Features of source rocks in the Xujiahe Formation at the transitional zone of central southern Sichuan Basin. Natural Gas Industry 29(6), 27-30 (in Chinese).

Zhao, W., Bian, C., Xu, C., Wang, H., Wang, T., Shi, Z., 2011. Assessment on gas accumulation potential and favorable plays within the Xu-1, 3 and 5 member of Xujiahe Formation in Sichuan Basin. Petroleum Exploration and Development 38(4), 385-393 (in Chinese).

Wu, X., Huang, S., Liao, F., Li, Z., 2011. Carbon isotopic compositions of coal-derived gas in the Xujiahe Formation and Jurassic in the Sichuan Basin. Petroleum Exploration and Development 38(4), 418-427.

Wu, X., Liu, Q., Zhu, J., Li, K., Liu, G., Chen, Y., Ni, C., 2017. Geochemical characteristics of tight gas and gas-source correlation in the Daniudi gas field, the Ordos Basin, China. Marine and Petroleum Geology 79, 421-425.

Yi, S., Lin, S., Yang, W., Shi, Z., Li, J., 2013. Condition of Xujiahe Formation large gas province formation in Sichuan Basin. Natural Gas Geoscience 24(1), 1-8 (in Chinese).

Zhao, W., Wang, H., Xu, C., Bian, C., Wang, Z., Gao, X., 2010. Reservoir-forming mechanism and enrichment conditions of the extensive Xujiahe Formation gas reservoirs, central Sichuan Basin. Petroleum Exploration and Development 37(2), 146-157 (in Chinese).

Zou Caineng, Tao Shizhen, Zhu Rukai, Yuan Xuanjun, Li Wei, Zhang Guangya, Zhang Xiang ang, Gao Xiaohui, Liu Liuhong, Xu Chunchun, Song Jiarong, Li Guohui, 2009. Formation and distribution of "continuous"'gas reservoirs and their giant gas province: A case from the Upper Triassic Xujiahe Formation giant gas province, Sichuan Basin. Petroleum Exploration and Development 36(3), 307-319 (in Chinese). 
